\title{
Free boundary problem for cell protrusion formations: theoretical and numerical aspects
}

\author{
Olivier Gallinato · Masahito Ohta • Clair Poignard • \\ Takashi Suzuki
}

the date of receipt and acceptance should be inserted later

\begin{abstract}
In this paper, a free boundary problem for cell protrusion formation is studied theoretically and numerically. The cell membrane is precisely described thanks to a level set function, whose motion is due to specific signalling pathways. The aim is to model the chemical interactions between the cell and its environment, in the process of invadopodia or pseudopodia formation. The model consists of Laplace equation with Dirichlet condition inside the cell coupled to Laplace equation with Neumann condition in the outer domain. The actin polymerization is accounted for as the gradient of the inner signal, which drives the motion of the interface. We prove the well-posedness of our free boundary problem under a sign condition on the datum. This criterion ensures the consistency of the model, and provides conditions to focus on for any enrichment of the model. We then propose a new first order Cartesian finite-difference method to solve the problem. We eventually exhibit the main biological features that can be accounted for by the model: the formation of thin and elongated protrusions as for invadopodia, or larger protrusion as for pseudopodia, depending on the source term in the equation. The model provides the theoretical and numerical grounds for single cell migration modeling, whose formulation is valid in $2 \mathrm{D}$ and $3 \mathrm{D}$. In particular, specific chemical reactions that occured at the cell membrane could be precisely described in forthcoming works.
\end{abstract}

Keywords Mathematical Biology, Cell Protrusion Formation, Free boundary problem, Finite differences on Cartesian grids

Mathematics Subject Classification (2000) 65M06, 65M12, 92C37

Olivier Gallinato

Team MONC, INRIA Bordeaux-Sud-Ouest, Institut de Mathématiques de Bordeaux, CNRS UMR 5251 \& Université de Bordeaux, 351 cours de la Libération, 33405 Talence Cedex, France

E-mail: olivier.gallinato@inria.fr

Masahito Ohta

Department of Mathematics, Tokyo University of Science, Tokyo, Japan

E-mail: mohta@rs.tus.ac.jp

Clair Poignard

Team MONC, INRIA Bordeaux-Sud-Ouest, Institut de Mathématiques de Bordeaux, CNRS UMR 5251 \& Université de Bordeaux, 351 cours de la Libération, 33405 Talence Cedex, France

E-mail: clair.poignard@inria.fr

Takashi Suzuki

Division of Mathematical Science, Osaka University, Osaka, Japan

E-mail: suzuki@sigmath.es.osaka-u.ac.jp 


\section{Introduction}

Invadopodia and pseudopodial protrusions are elongated shapes, which are formed during cell invasion and mesenchymal migration. These phenomena are the crucial points in the metastasic process, which is the major cause of death from cancer. From the biological point of view, both phenomena involve specific signaling pathways in the cell, that result from extracellular stimuli, as well as specific chemical interactions between the cell membrane and the extra-cellular matrix.

The aim of this paper is to present a general framework in which both phenomena can be modeled similarly, thanks to a free boundary model, which is valid for both 2 and 3 dimensions. From the modeling point of view, the model combines the interactions between the inner cell signaling and the extracellular matrix (ECM). These interactions occur across the cell membrane, which is precisely accounted for by a free boundary. The general philosophy of the modeling mimics the biological interactions: the cell production of chemical substances has an influence on the extracellular medium, whose changes are then detected by the cell itself, leading to the protrusion. The formation of the protrusion is a consequence of the displacement of the membrane, due to the gradient of the inner signal generated by the interactions between the membrane and the surrounding ECM. Therefore the velocity of the protrusion is not $a$ priori given but it is an unknown of the model. This is the novelty of the modeling, which complexifies dramatically both theoretical and numerical studies.

First we present our generic model, that holds in $2 \mathrm{D}$ and $3 \mathrm{D}$, and which is mathematically precise and relevant, and then we present an accurate finite difference method on Cartesian grid to solve accurately the problem. The well-posedness of our mode $\rrbracket^{1}$ is an important issue to address, since it provides a criterion to focus on to ensure a relevant modeling, and to prevent misinterpretation of the numerical simulations. The derivation of accurate scheme is also crucial since the model is highly nonlinear, and thus non accurate methods would lead to inconsistent results. Before stating the main results, we present the motivations and the biological features of cell migration that are accounted in this paper.

\subsection{Motivations and biological knowledge}

Early-stage carcinoma are mostly confined to epithelium, which is separated from the underlying tissue by a basement membrane composed of dense fibers of extracellular matrix. In order to cross this tight barrier, metastatic cells use a complex internal machinery, named invadopodia, which lies on the actin polymerization and that leads to the formation of proteolytic, protrusive and very localized subcellular structures. Once the basement membrane has been crossed, cells adopt a different migratory behavior to progress in the conjonctive tissue by projecting wider elongated protrusions. Cells migrating on 2D extracellular matrix form flat and plated structures called lamellipodia, at the leading edge, while cylindrical pseudopodia can be observed in 3D migration. Pseudopodia result also from both actin polymerization and cytoskeleton reorganization, as for invadopodia, but they grow as a response to a weak external gradient of chemoattractants, which are released by neighboring blood vessels. Thus such protrusions are wide directional migration structures, while invadopodia are local subcellular structures that are needed to perforate and pass through collagen walls. In Fig. 1 we present the different mechanisms that lead either to invadopodium or to pseudopod, and Fig. 2 presents the biological evidences of these protrusions, as described by [3,26,23]. We refer to [7] for a biological review of migration process.

In their review [11, Holmes and Edelstein-Keshet proposed a comparison of the different models for cell motility. Discrete cell automaton or hybrid models are well-designed to account for all the biological

\footnotetext{
1 As present hereafter, our model is somehow related to Hele-Shaw-type models: in Appendix A we show how wellposedness of the Hele-Shaw model can be tackled thanks to our approach.
} 


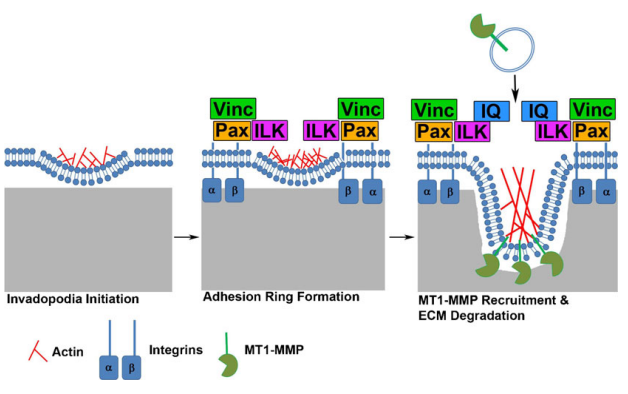

(a) Pathways for invadopodium formation 3

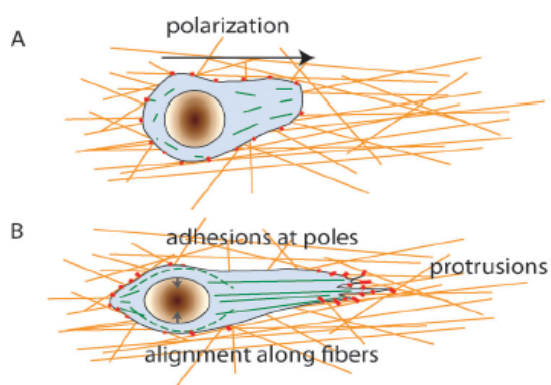

(b) Pathway for pseudopod formation 23

Fig. 1: Comparison of the mechanisms for invadopodium and pseudopodial protrusion.

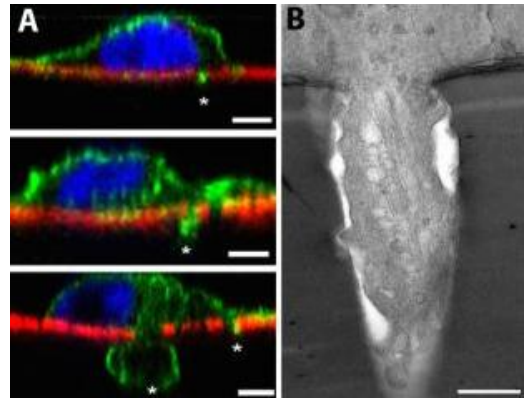

(a) Invadopodium formation 26
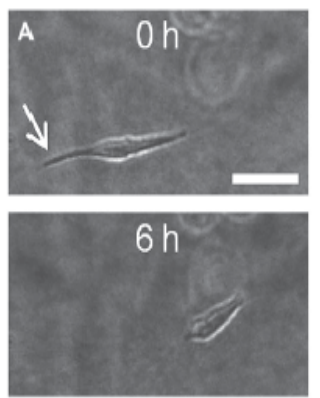

(b) 3D cell migration 23

Fig. 2: Comparison of the biological evidences of invadopodium and pseudopodial protrusions.

pathways involved in the protrusion formation. For invadopodia, one can cite the work of Weaver's group [5], while for cell motility and polarization one can cite papers of Edelstein-Keshet's group [19, 18, 11]. However these models are limited by their computational cost, which increases dramatically with the refinements of spatial and time discretizations. In addition they usually involve a large number of hardly measurable parameters, which prevent any sensitivity analysis of the model. Moreover the numerical stability of the simulations can be hardly shown. For all these reasons, we prefer dealing with continuous models based on partial differential equations (PDEs), in order to describe the phenomena at the cell scale. The biological knowledge of the molecular interactions can be accounted for either thanks to multiscale approaches or by a coupling with ad hoc phenomenological models that translate at the cell scale the molecular phenomena. Such perspectives are very challenging and very relevant from the modeling point of view, however it is necessary to derive first a generic PDE model at the cell scale.

Among the 2D/3D spatial PDE models, phase field method provides diffuse description of the cell membrane. The actin concentration $\phi$ satisfies a reaction-diffusion equation, and the membrane is implicitly located in the region where the gradient of $\phi$ is large. Levine and Rappel [16] use this approach for cell chemotaxis. In parallel, Saitou et al. derived such a kind of models for the formation and the maturation of invadopodia [25. They describe the distribution of specific enzymes, called Matrix Metalloproteinases (MMPs), which degrade the extracellular matrix, creating then ligands. These ligands, when bound to the membrane receptors, generate a signal which polymerizes the actin, leading to the formation of the invadopodium. Figure 3 provides the schematic diagram and the numerical results provided by Saitou et al. [25]. As one can see on 3(b), the main drawbacks of such modeling lie in diffusive location of the membrane. In particular, specific membrane receptors and ion channels such as $\mathrm{Na}^{+} / \mathrm{H}^{+}$exchangers or sodium channels that might be crucial in cell migration - see Stock and Schwab 28] and Yang et al. 32] 


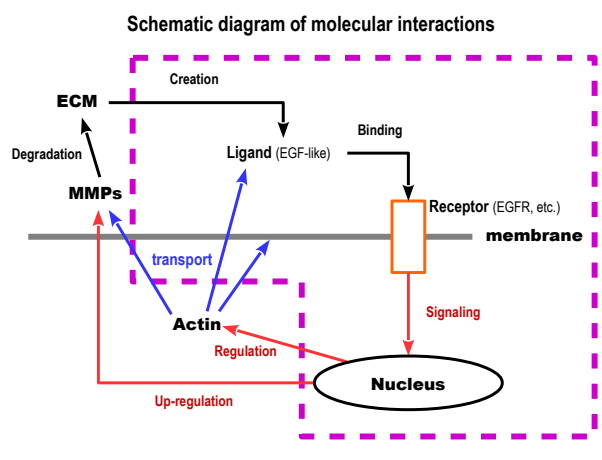

(a) Molecular interactions involved in the invadopodia model of Saitou et al. The dashed line shows the processes we focus on in this article.

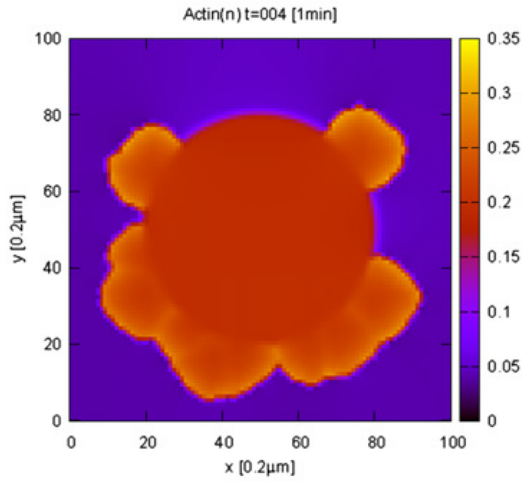

(b) Numerical formation of invadopodium by Saitou et al. 25.

Fig. 3: Invadopodia model of Saitou et al. 25].

- cannot be considered. Moreover, in the numerical simulations, the cell shape, and thus the protrusions, depend arbitrarily on the numerical criterion for actin localization.

Level-set methods are thus preferred to account precisely for membrane displacement. Strychalski et al. propose in 29] a finite volume method to solve a reaction-diffusion equation in moving geometries. Roughly speaking, given a velocity field $U$, which drives the cell membrane, they give a numerical scheme to account for the biochemical reaction-diffusion in the moving cell. Thanks to biomechanical considerations, Herant and Dembo use a two-phase fluid to describe the cell migration in the extracellular network [10]. Each phase of the cell (the cytoskeleton and cytosol) is driven at a specific velocity and the stress tensor due to the interactions between the network and the cell membrane is imposed in order to drive the protrusion. Wolgemuth and colleagues propose 4 different models based on moving boundary for cell crawling on flat surface (see [31]). Assuming that the whole cell is driven by a given velocity, they investigate 4 mechanisms for the limitation of the protrusion velocity (myosin contraction-driven motility, G-actin transport-limited motility, Rac/Rho-regulated motility and a model describing the limitation of the rate of protrusion by microtubule-based transport of vesicles to the leading edge limits).

These previous level-set based models provide sharp localization of the membrane, however for each model, the velocity of the protrusion is somehow imposed: Strychalski et al. 29] impose a global velocity field $U$, which drives the cell, while Herant and Dembo [10] impose the normal component of the velocity on different part of the cell membrane. Wolgemuth et al. 31 impose a directional velocity on the cell membrane, which is weighted by myosin stress. On the other hand biologists have shown that the protrusion formation results from interactions between the inner cell and the surrounding ECM. For instance Weaver's group has demonstrated the role of ECM rigidity, as well as the spacing of collagen fibers on invadopodia formation [2,, 5 . In addition to these papers, it has been demonstrated by the same group in 12 that MT1-MMPs play a crucial role in degrading ECM and then promote invadopodia. For migratory behaviors, Ridley et al. 24] demonstrate the way cells detect and amplify external chemical gradient thanks to proteins localized on the cell membrane.

Therefore there are biological evidences that protrusion formation (and thus the velocity of the protrusion) results from a response to chemical pathways between cell membrane and external stimuli. The present paper aims at providing the grounds for a cell modeling that describes the membrane motion through interactions between the cell and its microenvironment. Importantly, the velocity of the protrusion is an unknown quantity of the model, unlike the previously cited works. This novelty 
complexifies drastically the theoretical and the numerical studies. In particular, the well-posedness is not trivial, and inconsistant numerical schemes would lead to irrelevant conclusions.

It is worth noting that our goal is to provide a core model, and to study it theoretically and numerically. Therefore we only deal with simple phenomenological description of invadopodium and pseudopodium formation, which will be enriched by more biological features in following research. In the case of invadopodia, MMP enzymes are embedded in the cell membrane and degrade the extracellular matrix by contact, creating a ligand flux, while for pseudopodia, the source of chemoattractant diffuses far from the cell membrane. For the sake of simplicity, and since we are mainly concerned in the membrane description, we do not consider the production of MMPs: we assume that the ligand is created by a local concentration of MMP, which is a given Gaussian function on the interface. In the case of pseudopodial structures, chemoattractants are diffusing from a neighboring blood vessel and create an external gradient that leads to the cell polarization: proteins that are involved in actin polymerization are advected towards the leading edge of the cell. Thus, pseudopodium-like protrusion modeling needs to take the protein localization into account. Introducing a function for protein localization, and modifying the datum, one model pseudopodium formation in a similar way as for invadopodium. In each biological situation, more realistic complexification of the model will be addressed in forthcoming works, without changing the main structure of the model.

\subsection{Simple model for protrusion formation and main results}

Let us present our model, that describes the protrusions formation process. Interestingly, we show that thanks to slight modifications, one can pass from invadopodia to pseudopodial protrusions. We emphasize that we do not account for the membrane detachment phenomena which will lead to cell migration: only the first step of protrusion formation is accounted for.

\subsubsection{Invadopodium formation}

We first consider the invadopodium formation. The signaling pathways is a simplified version of Saitou et al. It is schematized by the scheme of Figure 4(a), while the geometrical frameworks is detailed in Figure 4(b). At any time $t$, the cell, denoted by $\mathcal{O}_{t}^{i}$ is embedded in the domain $\Omega$. The ECM is the domain

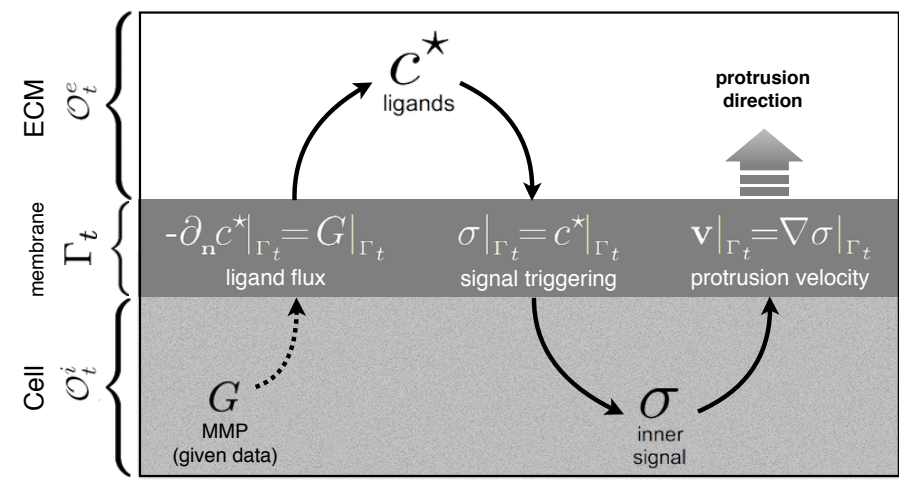

(a) Schematic diagram of the molecular interactions for invadopodia

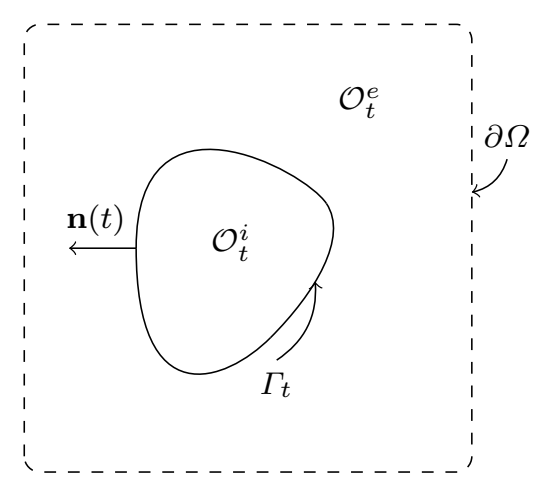

(b) Geometrical setting.

Fig. 4: Schematic diagram of the molecular interactions involved in our model and geometrical settings. The cell $\mathcal{O}_{t}^{i}$ is imbedded in the bath $\mathcal{O}_{t}^{e}$. The whole domain $\Omega$ does not depend on the time variable. It is defined by $\Omega=\mathcal{O}_{t}^{e} \cup \overline{\mathcal{O}_{t}^{i}}$

out of the cell, denoted by $\mathcal{O}_{t}^{e}$. At any time, the cell membrane $\Gamma_{t}$ is parameterized by the function $\gamma(t, \cdot)$ 
defined on the torus $\mathbb{T}=\mathbb{R} / 2 \pi \mathbb{Z}$. Assume the flux of MT1-MMP enzymes $g(t, \cdot)$ be given at any time on the cell membrane. It generates a flux of degraded matrix (called ligands and denoted by $c^{\star}$ ) on the cell boundary, and these ligand $\$^{2}$ diffuse in the extracellular medium as described by equations (1a) $-(1 \mathrm{~b})$. When bound to the cell membrane, the ligands generate a signal $\sigma$, which diffuses inside the cell, as accounted for in equations $1 \mathrm{c}-1 \mathrm{~d}]$. The membrane is then transported by the normal velocity at the interface $1 \mathrm{e}$.

Degradation of the ECM:

$$
\begin{aligned}
& \Delta c^{\star}=0, \quad x \in \mathcal{O}_{t}^{e}, \\
& \left.c^{\star}\right|_{\partial \Omega}=0, \quad-\left.\partial_{\mathbf{n}} c^{\star}\right|_{\Gamma_{t}}=\left.g\right|_{\Gamma_{t}} .
\end{aligned}
$$

Generation of the inner signal for actin polymerization:

$$
\begin{aligned}
& \Delta \sigma=0, \quad t \in[0, T], \quad x \in \mathcal{O}_{t}^{i}, \\
& \left.\sigma\right|_{\Gamma_{t}}=\left.c^{\star}\right|_{\Gamma_{t}} .
\end{aligned}
$$

Motion of the cell membrane:

$$
\begin{aligned}
& \partial_{t} \gamma(t, \theta)=\nabla \sigma(\gamma(t, \theta)), \quad \theta \in \mathbb{T}, \\
& \Gamma_{t}=\{\gamma(t, \theta), \quad \theta \in \mathbb{T}\} .
\end{aligned}
$$

The actin polymerization is accounted for by the gradient of the inner signal: this is a very simple model for polymerization, which is a kind of linearized version of more complex models: the new polymerized actin is seen as a vector-field, which pushes the membrane. Note that we omit the time derivative for both ligands and signal diffusion, assuming that the dynamics are much faster than the characteristic time for protrusion formation. More precise models, that involve the production of MMP enzymes and other complex biological phenomena such as precise actin polymerization, as modeled by Mogilner 20. for instance, are not addressed in this paper. The new insight of the modeling lies in the description of the membrane velocity, which results from the equation and is not imposed as in previous papers [10,29, 31.

\subsubsection{Similar model for pseudopodial protrusion}

Model (1) holds for invadopodia formation, since the MT1-MMPs on the membrane generate an outer flux of ligands. For pseudopodia formation, the chemoattractant diffuses far from the membrane, and the proteins that amplify the signal are localized at the front of the cell, through a complex chemical process described by Ridley et al. in 24]. Now $c^{\star}$ denotes a chemoattractant, whose source, denoted by $g$, is located far from the membrane. Zero flux of the chemoattractant on the membrane is imposed, assuming that the substance does no enter into the cell. Therefore the boundary conditions (1b) are replaced by equality (2a). In the extracellular medium, the chemoattractant diffuses so (1a) still holds. To account for the proteins that amplify the cell sensing, we introduce a smooth function $\kappa$, such that the advection equation is given by (2b). To summarize, the modifications are just to replace equations $1 \mathrm{bb}$ and $1 \mathrm{e}$ by $2 \mathrm{a}$ and $2 \mathrm{~b}$ respectively.

$$
\begin{aligned}
& \left.c^{\star}\right|_{\partial \Omega}=g,\left.\quad \partial_{\mathbf{n}} c^{\star}\right|_{\Gamma_{t}}=0, \\
& \partial_{t} \gamma(t, \theta)=\kappa(\gamma(t, \theta)) \nabla \sigma(\gamma(t, \theta)), \quad \theta \in \mathbb{T},
\end{aligned}
$$

${ }^{2}$ We assume that MT1-MMPs are located in the vincinity of the membrane, and thus no ligand are created far from the cell, as described by the Dirichlet condition on $\partial \Omega$. 


\subsection{Outline of the paper}

It is important, for theoretical but also modeling considerations to exhibit criteria for which the above models are well-posed. Such criteria will ensure the consistency of the modeling, otherwise ill-posedness would lead to irrevelant interpretations of the numerical results. For the same reason, accurate numerical schemes have to be developed, since the model is highly non linear. For explicitly given velocity, such issues are much easier to solve. Here, the main difficulties from both theoretical and numerical point of view lies in the fact that the velocity of the cell membrane is driven by the gradient of the signal $\sigma$.

In the next Section 22 we present the main results of the paper, in terms of biological and mathematical insights. We then present in Section 3 the main arguments that lead to well-posedness. For the sake of simplicty, we focus on the 2D case. Note that the difficulty lies in the fact that two domains are involved (the cell cytoplasm and the extracellular medium) and the velocity is the gradient of the inner chemical signal. As usual for such free-boundary problems [13,33, the proof for well-posedness is based on appropriate quasilinearization. Interestingly, this leads to a parabolic type equation that prevents the a priori loss of regularity. More precisely, thanks to Lagrangian formalism and complex analysis tools we provide the Dirichlet-to-Neumann operators in both inner and outer domains that make it possible to rewrite equivalently the free boundary problem on the torus $\mathbb{T}$ as provided in Lemma 10 . The quasilinearization is presented in Subsection 3.2, leading to the well-posedness result under a sign condition of the datum $g$. The sign condition under which holds the well-posedness is a kind of Taylor criterion ${ }^{3}$ adapted to our model. Section 4 is devoted to present our first order finite difference method on Cartesian grid, which ensures the accuracy of the simulations. The numerical method relies on immersed boundary and ghost fluid methods. We introduce a new continuous stencil for the gradients at the interface -the cell membrane- which stabilizes the standard schemes such as this of Cisternino and Weynans 4. We then illustrate numerically that instabilities appear if the positivity of $g$ is not satisfied and we show numerically the protrusions formation, similarly to biological images. Interestingly, numerical simulations for invadopodium and pseudopodial protrusions, are qualitatively similar to the biological observations. In Appendix A we show how the well-posedness of the Hele-Shaw model (also called Muskat model) can be tackled thanks to our approach.

\section{Main results of the paper and perspectives}

The main results of this paper are threefold:

- From the modeling point of view, we have derived a simple model for cell protrusion formation for which the protrusion velocity is not imposed. The velocity results from the interactions between the cell and the ECM. The actin polymerization is accounted for by the gradient of the inner signal. Interestingly, changing the interactions between the cell and the ECM lead to different protrusions, as explained in subsection 2.1. More precisely, for invadopodia, the source of the extracellular signal is located near the interface, leading to sharp protrusions, while for pseupodia, the chemical source is located far from the cell, and the signal is amplified by membrane receptors, leading to larger protrusions.

- We prove the well-posedness of the free boundary problem in Sobolev spaces, as stated in subsection 2.2. This theoretical result ensures the consistency of the model. Since the velocity of the cell membrane derives from the gradient of the signal, well-posedness is not trivial. The proof is performed in a bidimensional framework, which contains all the key points and benefits from complex analysis tools.

\footnotetext{
${ }^{3}$ In water waves 1533 13, Taylor criterion is a criterion under which instabilities occur.
} 
- We provide a first order finite difference method to solve the problem based on immersed boundary and ghost fluid methods. We propose a new continuous stencil for the gradient discretization at the interface which stabilizes the standard schemes. Interestingly, such stabilization makes it possible long time simulations, providing consistent numerical results.

\subsection{Numerical simulations illustrating biological phenomena}

A plot of an invadopodium simulation is provided in Figure 5. Ligand accumulation (dark grey) is observed around the membrane area where MMP enzymes are concentrated (Fig. 5(b)]. Consequently, the signal accumulates in the adjacent cytoplasmic area (Fig. 5(c)], and leads to the actin polymerization and thus invadopodium growth. Interestingly, the actin polymerization is concentrated in the

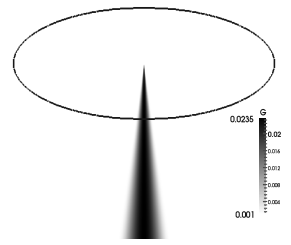

(a) Initial cell shape and initial lifting of MMP distribution.

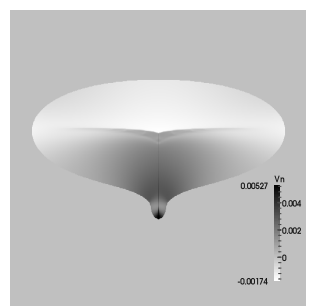

(d) Normal velocity, which reflects actin polymerization.

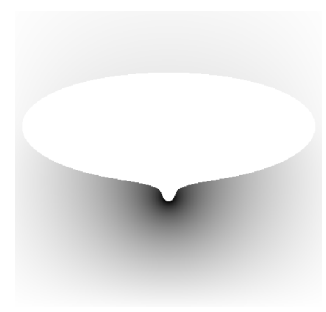

(b) Ligand diffusion after invadopodium formation.

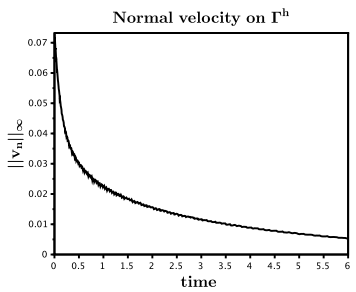

(e) Protrusion stabilization

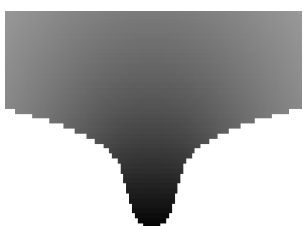

(c) Focus on the invadopodium.

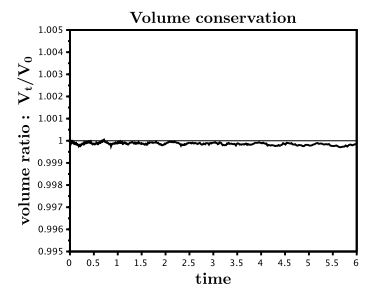

(f) Cell volume conservation along the simulation.

Fig. 5: Numerical invadopodium formation. Fig. 5(b) provides the ligand distribution, Fig. 5(c) is a zoom of the inner signal distribution. Fig. $5(\mathrm{f})$ shows the consistency of the numerics since the variations in volume are less than $0.05 \%$. In Fig. 5(d) the dark areas are for outcoming velocity and thus the actin polymerization, the light areas are for incoming velocity. As observed in the experiments, the velocity decreases, leading to a stabilization of the protrusion.

protrusion, as described by Branch et al. 3. The protrusion velocity decreases, indicating a tendency to invadopodium stabilization (Fig. $5(\mathrm{e})$. We also verified the numerical volume conservation ${ }^{4}$, which illustrates the accuracy of the numerical scheme.

Figure 6 shows a simulation of a pseudopodial projection, at the leading edge of the cell, in response to a chemotactic signal which is diffused from the right boundary of the domain.

Fig. 5 and Fig. 6 give an idea of the various changes in morphology of metastatic cancer cell during the invasion and migration, as accounted for by our model. Note only the protrusion formations are accounted for here. Critical processes involved in migration (binding to collagen fibers at the front of the pseudopodial protrusion, myosin-dependent retraction of the cell rear) are not considered in this study.

\footnotetext{
4 Note that volume conservation is not clear, from the biological point of view, but it results from our modeling of invadopodia, since the velocity of the interface is divergent free.
} 


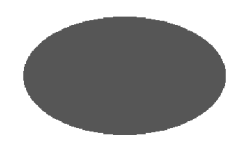

(a) Initial cell shape.

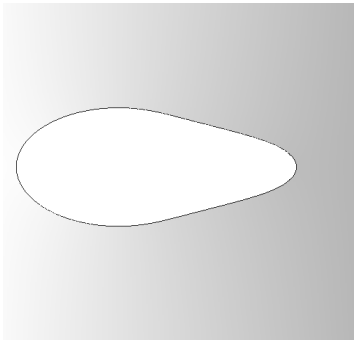

(b) Extracellular chemical signal distribution

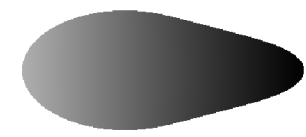

(c) Inner signal distribution after pseudopod formation.

Fig. 6: Simulation of a pseudopodial projection. Fig. 6(b) provides the distribution of the extracellular chemoattractant. The gradient is quite low, but it is amplified by the membrane proteins described by $\kappa$ in equation $2 \mathrm{~b}$, which leads the formation of a large protrusion.

2.2 Well-posedness under specific sign condition

From the theoretical point of view, we first state that our free boundary problem is well-posed under a specific sign condition on the data. For the sake of simplicity we focus on the 2-dimensional framework, which makes it possible to use simple tools of complex analysis. We are confident that similar results hold in 3D, but the analytical tools are more complex to deal with and this is far beyond the scope of the present paper. Our well-posedness result is stated in Lagrangian formalism, which easier to deal with.

We supposed that the initial location of the cell membrane $\Gamma_{0}$ is a perturbation of the unit circle parameterized by $\gamma_{0}$ :

$$
\gamma_{0}=e^{i \theta}+\xi_{0}(\theta)
$$

At any time $t, \Gamma_{t}$ is supposed to be parameterized by the counter-clockwisely oriented vector-field $\gamma$ :

$$
\Gamma_{t}=\left\{\gamma(t, \theta):=e^{i \theta}+\xi(t, \theta), \quad \theta \in[0,2 \pi]\right\}
$$

Theorem 1 (Well-posedness of the problem). Let $\Omega$ be a smooth domain of $\mathbb{R}^{2}$ which strictly contains the unit disk, and denote by $\Gamma_{0}$ the initial location of the membrane, given as a perturbation of the unit circle : $\Gamma_{0}=\left\{\gamma_{0}(\theta)=e^{i \theta}+\xi_{0}(\theta), \theta \in \mathbb{T}\right\}$.

Let $s \geq 3$, and let $g \in W^{1, \infty}\left(\mathbb{R}^{+} ; H^{s+1 / 2}(\Omega)\right)$ be such that for a given $\alpha>0$,

$$
g(t, x) \geq \alpha>0
$$

There exist $M>0$ and $T>0$ small enough such that if

$$
\left\|\xi_{0}\right\|_{H^{s}(\mathbb{T})} \leq M
$$

then, there exists a unique solution $\left(\gamma, c^{\star}, \sigma\right)$ on $(0, T)$ to problem (1) such that

$$
\gamma \in L^{\infty}\left(0, T ; H^{s}(\mathbb{T})\right) \cap L^{2}\left(0, T ; H^{s+1}(\mathbb{T})\right),
$$

and for almost any $t \in(0, T)$,

$$
c^{\star} \in H^{s+1 / 2}\left(\mathcal{O}_{t}^{e}\right), \quad \sigma \in H^{s+1 / 2}\left(\mathcal{O}_{t}^{i}\right) .
$$

The above theorem ensures that as soon as the data $g$ is positive, the model is well-posed. Therefore any enrichment of the model, for instance any model from which $g$ could result should focus on such positivity. 
Remark 2 (On the assumption of small data and the bidimensional framework). We focus on the bidimensional framework, which contains the main idea of the proof, but benefits from the convenient tools of complex analysis.

Nalimov, Yosihara, and Iguchi [21,33, 13] uses such tools for water waves well-posedness. Our model is very different since two domains are involved and the fluxes are discontinuous across the membrane. Moreover the quasilinearized problem from which the well-posedness results is also different, but the spirit of the proofs is similar. We are also confident that the result holds in $3 D$ thanks to more complex tools, but this is not the aim of the paper.

\subsection{Perspectives}

Even simple from the biological point of view, since the signaling pathways are drastically simplified, we propose in this paper a PDE system which is relevant for the protrusion formation. In particular, the free-boundary problem makes it possible to localize precisely the membrane, and the velocity of the protrusion formation is not imposed but it is an unknown of the PDE system. Our numerical scheme is accurate and stable, allowing long time accurate simulations. Interestingly, we exhibit decreasing of the velocity of the protrusion, leading to its stabilization as observed in the experiments. More biological phenomena will be accounted for in forthcoming works. In the case of invadopodia, cytoplasmic and membrane dynamics of MMPs remain to be explored to obtain a realistic time-dependent function $g$, in order to calibrate the model with biological data. The issue of the divergence-free velocity also is still unclear. It is also important to note that we focus in this paper on the protrusion formation: the detachment process, that would lead to the cell migration is not accounted for here, and the model will be completed in forthcoming works.

\section{Mathematical analysis}

In order to prove the well-posedness of Problem (1), we apply the strategy developed by Yosihara and Iguchi in [33,13, for water-waves to our problem, which is somehow more complex since it involves two phases unlike the standard water-waves problem. More precisely, using the Lagrangian form of Problem (1), we prove the well-posedness for small data around the unit circle, meaning that the free boundary $\gamma(t)$ for $t \in[0, T]$ is in a neighborhood of the unit circle. At $t=0, \Gamma_{0}$ is given as

$$
\Gamma_{0}=\left\{\gamma_{0}(\theta):=e^{i \theta}+\xi_{0}(\theta), \quad \theta \in[0,2 \pi]\right\} .
$$

and for any $t \in(0, T), \Gamma_{t}$ is parameterized as

$$
\Gamma_{t}=\left\{\gamma(t, \theta):=e^{i \theta}+\xi(t, \theta), \quad \theta \in[0,2 \pi]\right\} .
$$

The sketch of the proof is based on finding the non linear differential system satisfied by the components of $\xi$. It is worth noting that well-posedness is not trivial. Naive considerations make appear an $a$ priori loss of regularity, due to the fact that the velocity is given as the gradient of $\sigma$. The main idea of the proof is to rewrite equivalently problem $(1 \mathrm{a})-1 \mathrm{~b}-1 \mathrm{c}-(1 \mathrm{~d})-1 \mathrm{e}$ as a non linear system involving only the component of $\gamma$.

Remark 3 (Sketch of the proof). Differentiating (1d) with respect to $\theta$ and using (1e) on one hand, and on the other hand the Neumann condition (1b) on $c^{\star}$, lead to explicit expression (33) of $\left.\nabla c^{\star}\right|_{\gamma}$. Using the quasi Dirichlet-to-Neumann maps given in Lemma 6, which links the quasi tangential component of $\left.\nabla c^{\star}\right|_{\gamma}$ and to $\left.\nabla \sigma\right|_{\gamma}$ their respective quasi normal components, we obtain in Lemma 10 the non linear 
system (29)-30) satisfied by the components of $\xi$. Then we perform an appropriate quasilinearization of the non linear system in subsection 3.2. Our quasilinearization leads to equation (36), which can written on the torus as

$$
\partial_{t} W+2 g \frac{1}{1+R_{0}^{-2|D|}} \mathcal{H} \partial_{\theta} W=\text { Source term } .
$$

Using the fact that the symbol of $\mathcal{H} \partial_{\theta}$ is $|k|$, we then infer that if $g$ is positive and bounded away from zero, $W$ satisfies a parabolic equation, which prevents the a priori loss of regularity, leading thus to the well-posedness.

This section is split into 2 subsections. We first present the quasi Dirichlet-to-Neumann maps in subsection 3.1. which are useful to write equivalently the free-boundary problem on the torus. Then we perform the quasilinearization in subsection 3.2 and we conclude by the well-posedness.

We introduce the following useful notations:

Notation 4. To simplify notation, we denote by $\zeta_{0}$ the vector

$$
\zeta_{0}(\theta):=\left(\begin{array}{c}
\cos \theta \\
\sin \theta
\end{array}\right)=e^{i \theta}
$$

The outward normal $\mathbf{n}$ to $\Gamma$ is given by

$$
\mathbf{n}=\frac{1}{\left|\partial_{\theta} \gamma\right|} \partial_{\theta} \gamma^{\perp}, \quad \text { with } \quad \partial_{\theta} \gamma^{\perp}=\left(\begin{array}{c}
\partial_{\theta} \gamma_{2} \\
-\partial_{\theta} \gamma_{1}
\end{array}\right)=\partial_{\theta} \zeta_{0}^{\perp}+\partial_{\theta} \xi^{\perp} .
$$

For the sake of simplicity, we suppose that the outer boundary $\partial \Omega$ of $\Omega$ is the circle of radius $R_{0}>1$. As shown in [13], the following proof can be extended to other smooth geometries but this complexifies the calculations.

We generically denote by $L^{2}$ and $H^{s}$, for $s \geq 0$, the Lebesgue space $L^{2}(\mathbb{T})$ and the Sobolev space $H^{s}(\mathbb{T})$, respectively.

- For any $f \in L^{2}(\mathbb{T})$, we denote by $\hat{f}_{k}$ its $k^{\text {th }}$-Fourier coefficient defined by

$$
\hat{f}_{k}=\frac{1}{2 \pi} \int_{0}^{2 \pi} f(\theta) e^{-i k \theta} d \theta
$$

$-H^{s}$ is then defined by

$$
H^{s}=\left\{f \in L^{2}(\mathbb{T}), \quad \sum_{k \in \mathbb{Z}}\left(1+|k|^{2}\right)^{s}\left|\hat{f}_{k}\right|^{2}<+\infty\right\}
$$

- We denote by $\mathcal{P}_{0}$ the projection on the constants:

$$
\mathcal{P}_{0} f:=\frac{1}{2 \pi} \int_{0}^{2 \pi} f(\varphi) d \varphi=\hat{f}_{0}, \quad \forall f \in L^{2} .
$$

- The Hilbert transform $\mathcal{H}$ is the zeroth order operator defined as

$$
\mathcal{H} f:=\sum_{k \in \mathbb{Z}}(-i \operatorname{sgn}(k)) \hat{f}_{k} e^{i k \theta}, \quad \forall f \in L^{2} .
$$

- For $\lambda>0$, we denote by $R_{0}^{-\lambda|D|}$ the smoothing operator

$$
R_{0}^{-\lambda|D|} f:=\sum_{k \in \mathbb{Z}} R_{0}^{-\lambda|k|} \hat{f}_{k} e^{i k \theta}
$$


3.1 Quasi Dirichlet-to-Neumann maps and equivalent PDE system on $\Gamma_{0}$

We remind the definition of the functional spaces $L_{0}(r, s ; \tau)$ introduced by Yosihara (Definition 4.21 at page 70 of [33]):

Definition 5 (The spaces $L_{0}(r, s ; \tau)$ of Yosihara [33]). Let $s>0$ and $(r, \tau) \in[0, s]^{2}$.

We say that an operator $\mathcal{B}(\xi)$ depending on $\xi:=\left(\xi_{n}\right)_{n=1, \cdot, 4} \in\left(H^{s}\right)^{4}$ belongs to $L_{0}(r, s ; \tau)$ if there exist $C_{s}>0$ and $C_{\tau}>0$ such that the following two estimates hold

$$
\|\mathcal{B}(\xi) f\|_{s} \leq C_{s}\|\xi\|_{s}\|f\|_{r}, \quad \forall f \in H^{r}, \forall \xi \in\left(H^{s}\right)^{4} \text { s.t. }\|\xi\|_{s} \leq C_{s},\|\xi\|_{\tau} \leq C_{\tau}
$$

and for any $\left(\xi^{0}, \xi^{1}\right) \in\left(H^{s}\right)^{4} \times\left(H^{s}\right)^{4}$ such that $\left\|\xi^{j}\right\|_{\tau} \leq C_{\tau}$, and $\left\|\xi^{j}\right\|_{s} \leq C_{s}$, for $j=0,1$ :

$$
\left\|\mathcal{B}\left(\xi^{0}\right) f-\mathcal{B}\left(\xi^{1}\right) f\right\|_{s} \leq C_{s}\left\|\xi^{0}-\xi^{1}\right\|_{s}\|f\|_{r}, \quad \forall f \in H^{r} .
$$

The following lemma links the tangent and the normal components of the gradients of $c$ and $\sigma$, solutions at any time of the time-independent elliptic problems $1 \mathrm{ad}-1 \mathrm{~b}$ and $1 \mathrm{cc}-1 \mathrm{~d}$ ) respectively. Therefore we fix the variable $t$ and omit it in the notations.

Lemma 6 (Linking the normal component of the gradient to the tangent component). Let $s \geq 3$. Let $\gamma$ be defined by (3), with $\xi$ smooth enough. Let $c^{\star}$ and $\sigma$ be the solutions to (1a) $1 \mathrm{~b}$ and (1c) respectively.

There exist two $0^{\text {th }}$-order operators $\mathcal{L}_{\mathrm{i}}$ and $\mathcal{L}_{\mathrm{e}}$ such that5

$$
\begin{aligned}
{\left[\nabla \sigma(\gamma) \cdot \partial_{\theta} \zeta_{0}^{\perp}\right] } & =\mathcal{L}_{\mathrm{i}}(\xi)\left[\nabla \sigma(\gamma) \cdot \partial_{\theta} \zeta_{0}\right] \\
{\left[\nabla c^{\star}(\gamma) \cdot \partial_{\theta} \zeta_{0}\right] } & =\mathcal{L}_{\mathrm{e}}(\xi)\left[\nabla c^{\star}(\gamma) \cdot \partial_{\theta} \zeta_{0}^{\perp}\right]
\end{aligned}
$$

These operators are defined as

$$
\begin{aligned}
\mathcal{L}_{\mathrm{i}}(\xi) & =\mathcal{H}+\mathcal{A}_{1}(\xi), \\
\mathcal{L}_{\mathrm{e}}(\xi) & =\frac{1-R_{0}^{-2|D|}}{1+R_{0}^{-2|D|}} \mathcal{H}+\mathcal{A}_{2}(\xi),
\end{aligned}
$$

where the operators $\mathcal{A}_{1}$ and $\mathcal{A}_{2}$ belong to $L_{0}(2, s ; 2)$.

Remark 7. For $\xi \equiv 0, \Gamma$ is the unit circle and simple calculations in Fourier series imply that the inner Dirichlet-to-Neumann and the outer Neumann-to-Dirichlet maps are nothing but $\mathcal{L}_{\mathrm{i}}=\mathcal{H}$ and $\mathcal{L}_{\mathrm{e}}=\frac{1-R_{0}^{-2|D|}}{1+R_{0}^{-2|D|}} \mathcal{H}$ respectively. The above lemma generalizes these formula to a non-circular geometry.

Proof. The proof is adapted from Iguchi's paper [13. Define the function $z$ and $w$ as

$$
z(\theta)=\zeta_{0}(\theta)+\xi(\theta), \quad \text { and } \quad w(\theta)=R_{0} e^{i \theta} .
$$

We identify $\mathbb{R}^{2}$ and $\mathbb{C}$. Let $F_{\mathrm{i}}$ and $F_{\mathrm{e}}$ be defined by

$$
F_{\mathrm{i}}(z)=\partial_{x} \sigma(z)-i \partial_{y} \sigma(z), \quad F_{\mathrm{e}}(z)=\partial_{x} c^{\star}(z)-i \partial_{y} c^{\star}(z)
$$

and define the functions of the variable $\theta, f_{\mathrm{i}}, f_{\mathrm{e}}$ and $g_{\mathrm{e}}$, as

$$
f_{\mathrm{i}}(\theta)=F_{\mathrm{i}}(z(\theta)), \quad f_{\mathrm{e}}(\theta)=F_{\mathrm{e}}(z(\theta)), \quad g_{\mathrm{e}}(\theta)=F_{\mathrm{e}}(w(\theta)) .
$$

\footnotetext{
${ }^{5}$ Observe that for $\xi=0, \Gamma$ is the unit circle and the relations 6 are consistent with a simple calculus in Fourier series.
} 
First remark that

$$
\begin{array}{r}
\Re\left(e^{i \theta} f_{\mathrm{i}}\right)=\left.\nabla \sigma\right|_{\gamma} \cdot \partial_{\theta} \zeta_{0}^{\perp}, \quad \Im\left(e^{i \theta} f_{\mathrm{i}}\right)=-\left.\nabla \sigma\right|_{\gamma} \cdot \partial_{\theta} \zeta_{0}, \\
\Re\left(e^{i \theta} f_{\mathrm{e}}\right)=\left.\nabla c^{\star}\right|_{\gamma} \cdot \partial_{\theta} \zeta_{0}^{\perp}, \quad \Im\left(e^{i \theta} f_{\mathrm{e}}\right)=-\left.\nabla c^{\star}\right|_{\gamma} \cdot \partial_{\theta} \zeta_{0},
\end{array}
$$

Observe also that the Dirichlet boundary condition $1 \mathrm{~b}$ on $c^{\star}$ implies that the tangent gradient of $c^{\star}$ vanishes on $2 \Omega^{6}$.

$$
\nabla c^{\star}\left(R_{0} e^{i \theta}\right) \cdot\left(\begin{array}{c}
-\sin \theta \\
\cos \theta
\end{array}\right)=0,
$$

hence

$$
\forall \theta \in[0,2 \pi] \backslash\{ \pm \pi / 2\}, \quad g_{\mathrm{e}}(\theta)=\left.\frac{e^{-i \theta}}{\cos \theta} \partial_{x} c^{\star}\right|_{R_{0} e^{i \theta}}, \quad \text { and }\left.\quad \partial_{x} c^{\star}\right|_{ \pm i R_{0}}=0 .
$$

Since $F_{\mathrm{i}}$ and $F_{\mathrm{e}}$ are holomorphic, for any $z_{0} \in \Gamma$ the Cauchy-Riemann formula implies

$$
\begin{aligned}
F_{\mathrm{i}}\left(z_{0}\right) & =\frac{1}{i \pi} \mathrm{p} \cdot \mathrm{v} \cdot \int_{\Gamma} \frac{F_{\mathrm{i}}(z)}{z-z_{0}} d z \\
-F_{\mathrm{e}}\left(z_{0}\right) & =\frac{1}{i \pi} \mathrm{p} \cdot \mathrm{v} \cdot \int_{\Gamma} \frac{F_{\mathrm{e}}(z)}{z-z_{0}} d z-\frac{1}{i \pi} \int_{\partial \Omega} \frac{F_{\mathrm{e}}(w)}{w-z_{0}} d w,
\end{aligned}
$$

and for any $w_{0} \in \partial \Omega$ we have

$$
F_{\mathrm{e}}\left(w_{0}\right)=\frac{1}{i \pi} \mathrm{p} \cdot \mathrm{v} \cdot \int_{\partial \Omega} \frac{F_{\mathrm{e}}(w)}{w-w_{0}} d w-\frac{1}{i \pi} \int_{\Gamma} \frac{F_{\mathrm{e}}(z)}{z-w_{0}} d z
$$

Writing $z_{0}=z(\theta)$ and $w_{0}=w(\theta)$, the above integrals read respectively, for any $\theta \in \mathbb{T}$,

$$
\begin{aligned}
f_{\mathrm{i}}(\theta) & =\frac{1}{i \pi} \mathrm{p} \cdot \mathrm{v} \cdot \int_{0}^{2 \pi} \frac{f_{\mathrm{i}}(\varphi)}{z(\varphi)-z(\theta)} \frac{d z}{d \varphi} d \varphi \\
-f_{\mathrm{e}}(\theta) & =\frac{1}{i \pi} \mathrm{p} \cdot \mathrm{v} \cdot \int_{0}^{2 \pi} \frac{f_{\mathrm{e}}(\varphi)}{z(\varphi)-z(\theta)} \frac{d z}{d \varphi} d \varphi-\frac{1}{i \pi} \int_{0}^{2 \pi} \frac{g_{\mathrm{e}}(\varphi)}{w(\varphi)-z(\theta)} \frac{d w}{d \varphi} d \varphi, \\
g_{\mathrm{e}}(\theta) & =\frac{1}{i \pi} \mathrm{p} \cdot \mathrm{v} \cdot \int_{0}^{2 \pi} \frac{g_{\mathrm{e}}(\varphi)}{w(\varphi)-w(\theta)} \frac{d w}{d \varphi} d \varphi-\frac{1}{i \pi} \int_{0}^{2 \pi} \frac{f_{\mathrm{e}}(\varphi)}{z(\varphi)-w(\theta)} \frac{d z}{d \varphi} d \varphi,
\end{aligned}
$$

According to (8), since $\zeta_{0}(\theta)=e^{i \theta}$, one has

$$
\begin{aligned}
& \frac{1}{z(\varphi)-z(\theta)} \frac{d z(\varphi)}{d \varphi}=\frac{i e^{i \varphi}}{e^{i \varphi}-e^{i \theta}}+\frac{\partial}{\partial \varphi} \log \left(\frac{e^{i \varphi}-e^{i \theta}+\xi(\varphi)-\xi(\theta)}{e^{i \varphi}-e^{i \theta}}\right) \\
& \frac{1}{w(\varphi)-z(\theta)} \frac{d w(\varphi)}{d \varphi}=\frac{i e^{i \varphi}}{e^{i \varphi}-R_{0}^{-1} e^{i \theta}}+\frac{\partial}{\partial \varphi} \log \left(\frac{R_{0} e^{i \varphi}-e^{i \theta}-\xi(\theta)}{R_{0} e^{i \varphi}-e^{i \theta}}\right) \\
& \frac{1}{z(\varphi)-w(\theta)} \frac{d z(\varphi)}{d \varphi}=\frac{i e^{i \varphi}}{e^{i \varphi}-R_{0} e^{i \theta}}+\frac{\partial}{\partial \varphi} \log \left(\frac{e^{i \varphi}+\xi(\varphi)-R_{0} e^{i \varphi}}{e^{i \varphi}-R_{0} e^{i \theta}}\right)
\end{aligned}
$$

and obviously,

$$
\frac{1}{w(\varphi)-w(\theta)} \frac{d w(\varphi)}{d \varphi}=\frac{i e^{i \varphi}}{e^{i \varphi}-e^{i \theta}} .
$$

${ }^{6}$ Note that Iguchi introduced the notation

$$
W_{\mathrm{e}}^{(r)}-i W_{\mathrm{e}}^{(\theta)}=g_{\mathrm{e}}(\theta) e^{i \theta}
$$

and thus equation 10 reads $W_{\mathrm{e}}^{(\theta)}=0$. If $\partial \Omega$ is a perturbation of a circle, $\partial \Omega=\left\{R_{0}(1+b(\theta)) e^{i \theta}, \theta \in \mathbb{T}\right\}$, then, due to homogeneous Dirichlet condition on $\partial \Omega$ we deduce $W_{\mathrm{e}}^{(\theta)}=\frac{b^{\prime}}{1+b} W_{\mathrm{e}}^{(r)}$. In 13 homogeneous Neuman condition is imposed, which leads to $W^{(r)}=\frac{b^{\prime}}{1+b} W^{(\theta)}$ as given by equation (4.2) page 534 . 
Note that the above decompositions are useful since for small $\xi$, the second terms of the right-hand sides are small, and thus the leading parts are driven by the first terms independent of $\xi$. Moreover, as mentioned by Iguchi, one has the following characterization of the leading integral operators, for any $f \in L^{2}(\mathbb{T}):$

$$
\begin{aligned}
& \frac{1}{i \pi} \text { p.v. } \int_{0}^{2 \pi} \frac{i e^{i \varphi}}{e^{i \varphi}-e^{i \theta}} f(\varphi) d \varphi=e^{-i \theta}\left(i \mathcal{H}-\mathcal{P}_{0}\right)\left[e^{i \theta} f\right] \\
& \frac{1}{i \pi} \int_{0}^{2 \pi} \frac{i e^{i \varphi}}{e^{i \varphi}-R_{0}^{-1} e^{i \theta}} f(\varphi) d \varphi=e^{-i \theta}\left(i \mathcal{H} R_{0}^{-|D|+1}+\left(R_{0}^{-|D|+1}-R_{0} \mathcal{P}_{0}\right)\right)\left[e^{i \theta} f\right] \\
& \frac{1}{i \pi} \int_{0}^{2 \pi} \frac{i e^{i \varphi}}{e^{i \varphi}-R_{0} e^{i \theta}} f(\varphi) d \varphi=e^{-i \theta}\left(i \mathcal{H} R_{0}^{-|D|-1}-\left(R_{0}^{-|D|-1}+R_{0}^{-1} \mathcal{P}_{0}\right)\right)\left[e^{i \theta} f\right] .
\end{aligned}
$$

To simplify the notations, we set

$$
\begin{aligned}
& \mathcal{J}_{0}=i \mathcal{H}-\mathcal{P}_{0}, \\
& \mathcal{K}_{0}=i \mathcal{H} R_{0}^{-|D|+1}+\left(R_{0}^{-|D|+1}-R_{0} \mathcal{P}_{0}\right)=\mathcal{K}_{0}^{\Re}+i \mathcal{K}_{0}^{\Im}, \\
& \mathcal{L}_{0}=i \mathcal{H} R_{0}^{-|D|-1}-\left(R_{0}^{-|D|-1}+R_{0}^{-1} \mathcal{P}_{0}\right)=\mathcal{L}_{0}^{\Re}+i \mathcal{L}_{0}^{\Im} .
\end{aligned}
$$

We also introduce the 3 integral operators with complex symbols:

$$
\mathcal{B}_{j}(\xi) f(\theta)=-\frac{1}{\pi} \int_{0}^{2 \pi} b_{j}(\varphi, \theta ; \xi) \partial_{\varphi} f(\varphi) d \varphi
$$

where $b_{j}$ are the complex-valued functions defined as

$$
\begin{aligned}
& b_{1}(\varphi, \theta ; \xi)=\log \left(\frac{e^{i \varphi}-e^{i \theta}+\xi(\varphi)-\xi(\theta)}{e^{i \varphi}-e^{i \theta}}\right), \\
& b_{2}(\varphi, \theta ; \xi)=\log \left(\frac{R_{0} e^{i \varphi}-e^{i \theta}-\xi(\theta)}{R_{0} e^{i \varphi}-e^{i \theta}}\right) \\
& b_{3}(\varphi, \theta ; \xi)=\log \left(\frac{e^{i \varphi}+\xi(\varphi)-R_{0} e^{i \varphi}}{e^{i \varphi}-R_{0} e^{i \theta}}\right) .
\end{aligned}
$$

Thanks to these operators, equalities (11) read now

$$
\begin{aligned}
f_{\mathrm{i}}(\theta) & =e^{-i \theta} \mathcal{J}_{0}\left[e^{i \theta} f_{\mathrm{i}}\right]+\left(\mathcal{B}_{1}(\xi) f_{\mathrm{i}}\right)(\theta), \\
-f_{\mathrm{e}}(\theta) & =e^{-i \theta} \mathcal{J}_{0}\left[e^{i \theta} f_{\mathrm{e}}\right]-e^{-i \theta} \mathcal{K}_{0}\left[e^{i \theta} g_{\mathrm{e}}\right]+\left(\mathcal{B}_{1}(\xi) f_{\mathrm{e}}\right)(\theta)-\left(\mathcal{B}_{2}(\xi) g_{\mathrm{e}}\right)(\theta), \\
g_{\mathrm{e}}(\theta) & =e^{-i \theta} \mathcal{J}_{0}\left[e^{i \theta} g_{\mathrm{e}}\right]-e^{-i \theta} \mathcal{L}_{0}\left[e^{i \theta} f_{\mathrm{e}}\right]+\left(\mathcal{B}_{1}(\xi) g_{\mathrm{e}}\right)(\theta)-\left(\mathcal{B}_{3}(\xi) f_{\mathrm{e}}\right)(\theta) .
\end{aligned}
$$

It is crucial noting that thanks to classical Sobolev embedding, and similarly to Lemma 5.11 page 549 of Iguchi's paper [13], for $k \in\{1,2,3\}$, the above operator $\mathcal{B}_{k}$ belongs to $L_{0}(2, s ; 2)$, for $s \geq 3$, which means that they satisfy (5) with $r=2, \tau=2$, for any $s \geq 3$.

According to $(5)$ the norm of $\mathcal{B}_{1}(\xi)$ as an operator on $H^{2}(\mathbb{T})$ is bounded by the norm of $\xi$ in $H^{s}(\mathbb{T})$. Therefore for $\xi$ small enough in $H^{s}$ the norm of $\Re\left(e^{i \theta} \mathcal{B}_{1}(\xi) e^{-i \theta}\right)$ is also small, bounded by $\|\xi\|_{H^{s}}$ and thus the operator $1+\mathcal{P}_{0}-\Re\left(e^{i \theta} \mathcal{B}_{1}(\xi) e^{-i \theta}\right)$ is a perturbation of $1+\mathcal{P}_{0}$ which is invertible. Thus $1+\mathcal{P}_{0}-$ $\Re\left(e^{i \theta} \mathcal{B}_{1}(\xi) e^{-i \theta}\right)$ is also invertible and one has

$$
\left(1+\mathcal{P}_{0}-\Re\left(e^{i \theta} \mathcal{B}_{1}(\xi) e^{-i \theta}\right)\right)^{-1}-\left(1-\frac{1}{2} \mathcal{P}_{0}\right) \in L_{0}(2, s ; 2), \quad \text { for any } s \geq 3 .
$$


Denote by

$$
\begin{aligned}
\mathcal{J}(\xi) & =\mathcal{J}_{0}+e^{i \theta} \mathcal{B}_{1}(\xi) e^{-i \theta} \\
\mathcal{K}(\xi) & =\mathcal{K}_{0}+e^{i \theta} \mathcal{B}_{2}(\xi) e^{-i \theta} \\
\mathcal{L}(\xi) & =\mathcal{L}_{0}+e^{i \theta} \mathcal{B}_{3}(\xi) e^{-i \theta}
\end{aligned}
$$

and define, for $n=1,2,3$ the real integral operators $\mathcal{A}_{n}^{\Re}(\xi)$ and $\mathcal{A}_{n}^{\Im}(\xi)$ by

$$
\mathcal{A}_{n}^{\Re}(\xi)+i \mathcal{A}_{n}^{\Im}(\xi)=e^{i \theta} \mathcal{B}_{n}(\xi) e^{-i \theta} .
$$

Noting that thanks to equality $10, e^{i \theta} g_{\mathrm{e}}$ is a real-valued function, multiplying (19c) by $e^{i \theta}$ and taking successively the real and the imaginary parts, we infer the relations between $g_{\mathrm{e}}$ and $f_{\mathrm{e}}$ :

$$
\begin{aligned}
\left(1+\mathcal{P}_{0}-\mathcal{A}_{1}^{\Re}\right) e^{i \theta} g_{\mathrm{e}} & =-\left(\mathcal{H} R^{-|D|-1}+\mathcal{A}_{3}^{\Im}\right) \nabla c \cdot \partial_{\theta} \zeta_{0} \\
& +\left(R_{0}^{-|D|-1}+R_{0}^{-1} \mathcal{P}_{0}+\mathcal{A}_{3}^{\Re}\right) \nabla c \cdot \partial_{\theta} \zeta_{0}^{\perp} \\
\left(\mathcal{H}+\mathcal{A}_{1}^{\Im}\right) e^{i \theta} g_{\mathrm{e}} & =\left(\mathcal{H} R^{-|D|-1}+\mathcal{A}_{3}^{\Im}\right) \nabla c \cdot \partial_{\theta} \zeta_{0}^{\perp} \\
& +\left(R_{0}^{-|D|-1}+R_{0}^{-1} \mathcal{P}_{0}+\mathcal{A}_{3}^{\Re}\right) \nabla c \cdot \partial_{\theta} \zeta_{0} .
\end{aligned}
$$

Thus we obtain

$$
\begin{aligned}
e^{i \theta} f_{\mathrm{i}}(\theta) & =\mathcal{J}(\xi)\left[e^{i \theta} f_{\mathrm{i}}\right](\theta), \\
-e^{i \theta} f_{\mathrm{e}}(\theta) & =\mathcal{J}(\xi)\left[e^{i \theta} f_{\mathrm{e}}\right](\theta)+\mathcal{K}(\xi)\{1-\Re(\mathcal{J}(\xi))\}^{-1}\left[\Re\left(\mathcal{L}(\xi)\left[e^{i \theta} f_{\mathrm{e}}\right]\right)\right] .
\end{aligned}
$$

Taking the real part of (24), using (9) leads to

$$
\left(1+\mathcal{P}_{0}-\mathcal{A}_{1}^{\Re}(\xi)\right)\left[\nabla \sigma(\gamma) \cdot \partial_{\theta} \zeta_{0}^{\perp}\right]=\left(\mathcal{H}+\mathcal{A}_{1}^{\Im}(\xi)\right)\left[\nabla \sigma(\gamma) \cdot \partial_{\theta} \zeta_{0}\right],
$$

from which we infer 6a by setting

$$
\mathcal{L}_{\mathrm{i}}(\xi)=\left(1+\mathcal{P}_{0}-\mathcal{A}_{1}^{\Re}(\xi)\right)^{-1}\left(\mathcal{H}+\mathcal{A}_{1}^{\Im}(\xi)\right)=\mathcal{H}+\mathcal{A}_{1}(\xi) .
$$

Similarly, using the definition of $\mathcal{K}_{0}$ and $\mathcal{L}_{0}$ and observing that

$$
\mathcal{H} \mathcal{P}_{0}=\mathcal{P}_{0} \mathcal{H}=0, \quad \mathcal{H} R_{0}^{-|D|}=R_{0}^{-|D|} \mathcal{H},
$$

by taking the imaginary part of $(25)$ and using $(23)$, we infer that there exists $\mathcal{A}_{2}(\xi)$ such that

$$
\left.\nabla c^{\star}\right|_{\gamma} \cdot \partial_{\theta} \zeta_{0}=\left.\left(\frac{1-R_{0}^{-2|D|}}{1+R_{0}^{-2|D|}} \mathcal{H}+\mathcal{A}_{2}(\xi)\right) \nabla c^{\star}\right|_{\gamma} \cdot \partial_{\theta} \zeta_{0}^{\perp} .
$$

It is clear that as the operators $\mathcal{B}_{k}$, for $k=1,2,3$, the real-valued operators $\mathcal{A}_{1}$ and $\mathcal{A}_{2}$ belong to $L_{0}(2, s ; 2)$, which ends the proof of Lemma 6 .

Remark 8. Note that taking the imaginary part of (24), using (9) leads to

$$
\left(1+\mathcal{P}_{0}-\mathcal{A}_{1}^{\Re}(\xi)\right)\left[\nabla \sigma(\gamma) \cdot \partial_{\theta} \zeta_{0}\right]=-\left(\mathcal{H}+\mathcal{A}_{1}^{\Im}(\xi)\right)\left[\nabla \sigma(\gamma) \cdot \partial_{\theta} \zeta_{0}^{\perp}\right] .
$$

Remark 9. Using the fact that $\partial_{t} \gamma=\partial_{t} \xi$, equation 1e and the above lemma imply that

$$
\partial_{t} \xi \cdot \partial_{\theta} \zeta_{0}^{\perp}=\mathcal{L}_{\mathrm{i}}(\xi)\left\{\partial_{t} \xi \cdot \partial_{\theta} \zeta_{0}\right\}
$$


We now rewrite the nonlinear system(1) in terms of $X_{1}$ and $X_{2}$ defined as

$$
X_{1}=\xi \cdot \partial_{\theta} \zeta_{0}^{\perp}, \quad X_{2}=\xi \cdot \partial_{\theta} \zeta_{0} .
$$

Using this change of variables, the following equalities hold

$$
\begin{aligned}
\partial_{t} \xi & =\partial_{t} X_{2} \partial_{\theta} \zeta_{0}+\partial_{t} X_{1} \partial_{\theta} \zeta_{0}^{\perp}, \\
\partial_{\theta} \xi & =\left(\partial_{\theta} X_{2}+X_{1}\right) \partial_{\theta} \zeta_{0}+\left(\partial_{\theta} X_{1}-X_{2}\right) \partial_{\theta} \zeta_{0}^{\perp}, \\
\partial_{\theta} \xi^{\perp} & =-\left(\partial_{\theta} X_{1}-X_{2}\right) \partial_{\theta} \zeta_{0}+\left(\partial_{\theta} X_{2}+X_{1}\right) \partial_{\theta} \zeta_{0}^{\perp} .
\end{aligned}
$$

Since $\partial_{\theta} \gamma=\partial_{\theta} \zeta_{0}+\partial_{\theta} \xi$, we show in the next lemma that the system can be rewritten in terms of $X_{1}$, $X_{2}$ and their derivatives.

Lemma 10 (Equivalent problems). Problem (1) is equivalent to the following nonlinear problem written in terms of $\left(X_{1}, X_{2}\right)$ :

$$
\begin{aligned}
& \partial_{t} X_{1}=\mathcal{L}_{\mathrm{i}}(\xi)\left\{\partial_{t} X_{2}\right\}, \\
& \frac{\partial_{t} \gamma \cdot \partial_{\theta} \gamma}{\left|\partial_{\theta} \gamma\right|^{2}}\left(1+X_{1}+\partial_{\theta} X_{2}\right)+\frac{g}{\left|\partial_{\theta} \gamma\right|}\left(\partial_{\theta} X_{1}-X_{2}\right) \\
& =\mathcal{L}_{\mathrm{e}}(\xi)\left\{\frac{\partial_{t} \gamma \cdot \partial_{\theta} \gamma}{\left|\partial_{\theta} \gamma\right|^{2}}\left(\partial_{\theta} X_{1}-X_{2}\right)-\frac{g}{\left|\partial_{\theta} \gamma\right|}\left(1+X_{1}+\partial_{\theta} X_{2}\right)\right\},
\end{aligned}
$$

where

$$
\begin{aligned}
& \left|\partial_{\theta} \gamma\right|=\sqrt{\left(1+\partial_{\theta} X_{2}+X_{1}\right)^{2}+\left(\partial_{\theta} X_{1}-X_{2}\right)^{2}} \\
& \partial_{t} \gamma \cdot \partial_{\theta} \gamma=\partial_{t} X_{2}\left(1+\partial_{\theta} X_{2}+X_{1}\right)+\partial_{t} X_{1}\left(\partial_{\theta} X_{1}-X_{2}\right) .
\end{aligned}
$$

Remark 11. We thus have reduced our free boundary problem to the equivalent nonlinear system (29)(30) on $\left(X_{1}, X_{2}\right)$ set in the domain $(0, T) \times \mathbb{T}$. The well-posedness of this equivalent problem will thus lead to the well-posedness of our free boundary problem. The advantage lies in the fact that the involved operators are now better understood thanks to Lemma 6 .

Proof. Equality (29) comes from Lemma 6 and Remark 9. Differentiating (1d) with respect to $\theta$, we have

$$
\partial_{\theta} \gamma \cdot \nabla \sigma(\gamma)=\partial_{\theta} \gamma \cdot \nabla c^{\star}(\gamma)
$$

and thus thanks to 1 we deduce:

$$
\partial_{\theta} \gamma \cdot \partial_{t} \gamma=\partial_{\theta} \gamma \cdot \nabla c^{\star}(\gamma)
$$

The second equality of $1 \mathrm{~b}$ reads then

$$
\left(\partial_{\theta} \gamma\right)^{\perp} \cdot \nabla c^{\star}(\gamma)=-\left|\partial_{\theta} \gamma\right| g(t, \gamma(t, \theta))
$$

Therefore we obtain the following expression of $\nabla c^{\star}(\gamma)$ :

$$
\nabla c^{\star}(\gamma)=\frac{1}{\left|\partial_{\theta} \gamma\right|^{2}}\left(\left(\partial_{t} \gamma \cdot \partial_{\theta} \gamma\right) \partial_{\theta} \gamma-g\left|\partial_{\theta} \gamma\right| \partial_{\theta} \gamma^{\perp}\right)
$$

Thanks to $6 \mathrm{~b}$ and using the equalities 28 we deduce equation 30 . 
3.2 Quasilinearization and well-posed for small data

Following [13, we quasilinearize the system $\left[29-(30)\right.$. Let $\mathbb{Y}=\left(Y_{i}\right)_{i=1,4}$ be defined by

$$
Y_{1}=X_{1}, \quad Y_{2}=X_{2}, \quad Y_{3}=\partial_{\theta} X_{1}-X_{2}, \quad Y_{4}=\partial_{\theta} X_{2}+X_{1},
$$

and set

$$
W=\partial_{t} X_{2}
$$

From 29, we get

$$
\begin{aligned}
& \partial_{t} Y_{1}=\mathcal{L}_{\mathrm{i}}(\mathbb{Y})\{W\}, \\
& \partial_{t} Y_{2}=W, \\
& \partial_{t} Y_{3}=\partial_{\theta} \mathcal{L}_{\mathrm{i}}(\mathbb{Y})\{W\}-W, \\
& \partial_{t} Y_{4}=\partial_{\theta} W+\mathcal{L}_{\mathrm{i}}(\mathbb{Y})\{W\} .
\end{aligned}
$$

The following lemma is straightforward.

Lemma 12. Let $s \geq 3$. Suppose that $W \in L^{2}\left(0, T ; H^{s+1}\right)$ and let $\mathbb{Y}_{0} \in\left(H^{s}(\mathbb{T})\right)^{4}$. Then the solution $\mathbb{Y}$ to (34) with the initial condition $\left.\mathbb{Y}\right|_{t=0}=\mathbb{Y}_{0}$ is such that

$$
\mathbb{Y} \in L^{\infty}\left(0, T ;\left(H^{s}(\mathbb{T})\right)^{4}\right)
$$

and

$$
\|\mathbb{Y}\|_{L^{\infty}\left(0, T ;\left(H^{s}(\mathbb{T})\right)^{4}\right)} \leq\left\|\mathbb{Y}_{0}\right\|_{\left(H^{s}(\mathbb{T})\right)^{4}}+C \sqrt{T}\|W\|_{L^{2}\left(0, T ; H^{s+1}(\mathbb{T})\right)} .
$$

Moreover, if $\left(W_{1}, W_{2}\right) \in\left(L^{2}\left(0, T ; H^{s+1}\right)\right)^{2}$ and $\mathbb{Y}_{0} \in\left(H^{s}(\mathbb{T})\right)^{4}$, then the corresponding solutions $\mathbb{Y}_{1}$ and $\mathbb{Y}_{2}$ satisfy:

$$
\left\|\mathbb{Y}_{1}-\mathbb{Y}_{2}\right\|_{L^{\infty}\left(0, T ;\left(H^{s}(\mathbb{T})\right)^{4}\right)} \leq C \sqrt{T}\left\|W_{1}-W_{2}\right\|_{L^{2}\left(0, T ; H^{s+1}(\mathbb{T})\right)} .
$$

It remains to obtain the nonlinear equation for $W$. Equation reads now

$$
\begin{aligned}
& F_{1}^{2}(\mathbb{Y}) W+F_{1}(\mathbb{Y}) F_{2}(\mathbb{Y}) \mathcal{L}_{\mathrm{i}}(\mathbb{Y})\{W\}+F_{2}(\mathbb{Y}) g(t, \gamma(t, \theta) \\
& =\mathcal{L}_{\mathrm{e}}(\mathbb{Y})\left\{F_{1}(\mathbb{Y}) F_{2}(\mathbb{Y}) W+F_{1}^{2}(\mathbb{Y}) \mathcal{L}_{\mathrm{i}}(\mathbb{Y}) W-F_{1}(\mathbb{Y}) g(t, \gamma(t, \theta)\},\right.
\end{aligned}
$$

where $F_{i}$ are given for $i=1,2$ by

$$
F_{1}(\mathbb{Y})=\frac{1+Y_{4}}{\sqrt{\left(1+Y_{4}\right)^{2}+Y_{3}^{2}}}, \quad F_{2}(\mathbb{Y})=\frac{Y_{3}}{\sqrt{\left(1+Y_{4}\right)^{2}+Y_{3}^{2}}} .
$$

Similarly to Yosihara's and Iguchi's papers, differentiating the above equation with respect to $t$, we infer the following equation for $W$ :

$$
\partial_{t} W+2 g \frac{1}{1+R_{0}^{-2|D|}} \mathcal{H} \partial_{\theta} W=f\left(g, \partial_{t} g, \mathbb{Y}, W, \partial_{\theta} W\right),
$$

where $f$ is such that there exists $C>0$ such that if $\mathbb{Y}$ and $g$ are small enough in $\left(L^{\infty}\left(0, T ; H^{s}(\mathbb{T})\right)\right)^{4}$, $W^{1, \infty}\left(0, T ; H^{s+1 / 2}(\Omega)\right)$ respectively, one has the following inequalities for any $W$ small enough in $L^{\infty}\left(0, T ; H^{s}\right) \cap$ $L^{2}\left(0, T ; H^{s+1}\right)$ :

$$
\begin{array}{r}
\left\|f\left(\cdots, \mathbb{Y}, W, \partial_{\theta} W\right)\right\|_{L^{2}\left(0, T ; H^{s}\right)} \leq C\left(\sqrt{T}\|W\|_{L^{\infty}\left(0, T ; H^{s}\right)}\right. \\
\left.+\|\mathbb{Y}\|_{\left(L^{\infty}\left(0, T ; H^{s}\right)\right)^{4}}\|W\|_{L^{2}\left(0, T ; H^{s+1}\right)}\right), \\
\left\|f\left(\cdots, W_{1}, \partial_{\theta} W_{1}\right)-f\left(\cdots, \mathbb{Y}, W_{2}, \partial_{\theta} W_{2}\right)\right\|_{L^{2}\left(0, T ; H^{s}\right)} \leq C\left(\sqrt{T}\left\|W_{1}-W_{2}\right\|_{L^{\infty}\left(0, T ; H^{s}\right)}\right. \\
\left.+\|\mathbb{Y}\|_{\left(L^{\infty}\left(0, T ; H^{s}\right)\right)^{4}}\left\|W_{1}-W_{2}\right\|_{L^{2}\left(0, T ; H^{s+1}\right)}\right),
\end{array}
$$


where the dots $\cdots$ hold for the variables $\left(g, \partial_{t} g, \mathbb{Y}\right)$. For the sake of conciseness, we do not make explicit the expression of $f$ in terms of $F_{1}$ and $F_{2}$, but as in Yosihara's and Iguchi's papers, this function has clearly the above properties.

The main difference with these works lies in the partial differential equation satisfied by $W:=\partial_{t} X_{2}$. Actually, neglecting the non-linear terms of (36) we observe that the equation is parabolic since the symbol of $\mathcal{H} \partial_{\theta}$ is

$$
\mathfrak{s}_{\mathcal{H} \partial_{\theta}}(k)=|k|, \quad k \in \mathbb{Z} .
$$

Moreover one can easily check that due to the hypotheses (4) on $g$ at Theorem 1 that we recall here:

$$
\begin{array}{ll}
g \in W^{1, \infty}\left(\mathbb{R}^{+} ; H^{s+1 / 2}(\Omega)\right), \quad\|g\|_{W^{1, \infty}\left(\mathbb{R}^{+} ; H^{s+1 / 2}(\Omega)\right)} \leq M, & \text { for a given } M>0, \\
\forall(t, x) \in \mathbb{R} \times \Omega, \quad g(t, x) \geq \alpha, & \text { for a given } \alpha>0,
\end{array}
$$

the time-dependent operator $\left(\mathcal{A}_{g}, H^{1}\right)$ defined as

$$
\mathcal{A}_{g}:(t, w) \in \mathbb{R}^{+} \times H^{1} \mapsto 2 g(t) \frac{1}{1+R_{0}^{-2|D|}} \mathcal{H} \partial_{\theta} w,
$$

is m-accretive in the sense of Kato [14 since it satisfies the following properties:

1. $D\left(\mathcal{A}_{g}\right)=\left\{u \in H^{1 / 2}: \quad \mathcal{H} \partial_{\theta} u \in L^{2}\right\} \subset L^{2}$ does not depend on the time $t$.

2. For almost any $t>0,\left(\mathcal{A}_{g}(t), H^{1}\right)$ is m-accretive. This easily comes from the fact that $\left\langle\mathcal{H} \partial_{\theta} w, w\right\rangle=$ $\left\|w-\int_{\mathbb{T}} w\right\|_{H^{1 / 2}}^{2}$, therefore for any $\lambda>0$, one has

$$
\left\|v+\lambda \mathcal{A}_{g}(t) v\right\|_{L^{2}}^{2} \geq\|v\|_{L^{2}}^{2}+4 \alpha\left\|v-\int_{\mathbb{T}} v\right\|_{H^{1 / 2}}^{2}+4 \alpha^{2}\left\|\partial_{\theta} v\right\|_{L^{2}}^{2} \geq\|v\|_{L^{2}}^{2} .
$$

Moreover for any $\lambda>0$, for any $f \in L^{2}$, the equation

$$
u+\lambda \mathcal{A}_{g}(t) u=f
$$

has a unique solution since the bilinear form

$$
a(u, v):=\int_{\mathbb{T}} u v d \theta+\lambda \int_{\mathbb{T}} \mathcal{A}_{g}(t) u v d \theta
$$

is continuous and coercive on $H^{1 / 2}$ thanks to [4], and thus Lax-Milgram lemma provides the existence and uniqueness of $u$ in $D\left(\mathcal{A}_{g}\right)$.

3. Finally, for almost any $(t, s) \in\left(\mathbb{R}^{+}\right)^{2}$ one has

$$
\mathcal{A}_{g}(t) v-\mathcal{A}_{g}(s) v=(g(t)-g(s)) \frac{2}{1+R_{0}^{-2|D|}} \mathcal{H} \partial_{\theta} v
$$

and thus once again thanks to (4), one has

$$
\left\|\mathcal{A}_{g}(t) v-\mathcal{A}_{g}(s) v\right\|_{L^{2}} \leq \frac{\|g\|_{W^{1, \infty}\left(\mathbb{R}^{+} ; H^{s+1 / 2}\right)}}{\alpha}|t-s|\left(1+\|v\|+\left\|\mathcal{A}_{g}(s) v\right\|\right) .
$$

Therefore the 3 conditions of Kato's paper 14 are satisfied and thus the time-dependent equation $\partial_{t} w+\mathcal{A}_{g}(t)(w)=f$ admits a unique solution for any $f \in L^{1}\left(0, T ; H^{1 / 2}\right)$.

In the following, we focus on the energy estimates in order to prove the well-posedness of the quasilinearized system, thanks to the use of a classical fixed point method. 
Lemma 13 (A priori energy estimates). Let $s \geq 3$ and $\alpha>0$. Suppose that there exists a $2 \pi$-periodic solution $W \in L^{\infty}\left(0, T ; H^{s+1 / 2}\right) \cap L^{2}\left(0, T ; H^{s+1}\right)$ satisfying equation (36) with the initial condition $\left.W\right|_{t=0}=W_{0}$. Then there exist $M>0$ and $T>0$ small enough such that if $W_{0} \in H^{s}(\mathbb{T})$ and if $\mathbb{Y}$ and $g$ satisfy

$$
\begin{aligned}
& \left\|W_{0}\right\|_{H^{s}} \leq M, \quad\|\mathbb{Y}\|_{L^{\infty}\left(0, T ; H^{s}\right)} \leq M, \\
& g \in W^{1, \infty}\left(0, T ; H^{s+1 / 2}\right): g(t, x) \geq \alpha, \forall(t, x) \in(0, T) \times \Omega,
\end{aligned}
$$

then the solution $W$ satisfies:

$$
\sup _{t \in(0, T)}\|W(t, \cdot)\|_{H^{s+1 / 2}}+\|W\|_{L^{2}\left(0, T ; H^{s+1}\right)} \leq M .
$$

Proof. The proof is standard, thanks to the embedding $H^{s} \hookrightarrow L^{\infty}$ for any $s>1 / 2$ and using the fact that

$$
\left\langle W, \mathcal{H} \partial_{\theta} W\right\rangle=\|W-\bar{W}\|_{H^{1 / 2}}^{2} .
$$

More precisely, multiplying first equation 36 by $W$ and integrating by part in the space variable lead to

$$
\begin{aligned}
\frac{1}{2} \frac{d}{d t}\|W\|_{L^{2}}^{2}+\alpha\|W-\bar{W}\|_{H^{1 / 2}}^{2} & \leq \frac{d}{d t}\|W\|_{L^{2}}^{2}+\int_{\mathbb{T}} g \frac{1}{1+R_{0}^{-2|D|}} W \mathcal{H} \partial_{\theta} W d \theta \\
& \leq \int_{\mathbb{T}}|f W| d \theta
\end{aligned}
$$

from which we infer that for $M$ and $T$ small enough,

$$
\sup _{t \in(0, T)}\|W(t, \cdot)\|_{L^{2}} \leq M, \quad\|W\|_{L^{2}\left(0, T ; H^{1 / 2}\right)} \leq M .
$$

Similarly, multiplying by $\mathcal{H} \partial_{\theta} W$ we infer that for $M$ small enough:

$$
\frac{1}{2} \frac{d}{d t}\|W-\bar{W}\|_{H^{1 / 2}}^{2}+\frac{1}{2} \alpha\|W-\bar{W}\|_{H^{1}}^{2} \leq \int\left|f \mathcal{H} \partial_{\theta} W\right| d \theta
$$

and thus we infer that for $M$ and $T$ small enough,

$$
\sup _{t \in(0, T)}\|W(t, \cdot)\|_{H^{1 / 2}} \leq M, \quad\|W\|_{L^{2}\left(0, T ; H^{1}\right)} \leq M .
$$

Deriving until the order $s$ the equation (36) with respect to $\theta$, and denoting by $Z:=\partial_{\theta}^{s} W$ we obtain

$$
\partial_{t} Z+2 g \frac{1}{1+R_{0}^{-2|D|}} \mathcal{H} \partial_{\theta} Z=G
$$

where the right hand side is in $L^{2}\left(0, T ; L^{2}\right)$ according to 37$)$. The estimations

$$
\begin{aligned}
\int_{0}^{T} \int_{\mathbb{T}}|G Z| d \theta d t & \leq\|G\|_{L^{2}(0, T ; L 2)}\|Z\|_{L^{2}\left(0, T ; L^{2}\right)}, \\
& \leq(M+\sqrt{T})\|Z\|_{L^{2}\left(0, T ; H^{1}\right)}^{2}
\end{aligned}
$$

and

$$
\begin{aligned}
\int_{0}^{T} \int_{\mathbb{T}}\left|G \mathcal{H} \partial_{\theta} Z\right| d \theta d t & \leq\|G\|_{L^{2}\left(0, T ; L^{2}\right)}\|Z\|_{L^{2}\left(0, T ; H^{1}\right)} \\
& \leq(M+\sqrt{T})\|Z\|_{L^{2}\left(0, T ; H^{1}\right)}^{2},
\end{aligned}
$$

imply that for $M$ and $T$ small enough,

$$
\sup _{t \in(0, T)}\|Z(t, \cdot)\|_{H^{1 / 2}} \leq M, \quad\|Z\|_{L^{2}\left(0, T ; H^{1}\right)} \leq M,
$$

which ends the proof. 
The contraction estimates are obtained in the similar manner.

Definition 14. Let $M>0$.

We denote by $\mathcal{V}_{M}$ the subspace of $L^{\infty}\left(0, T ; H^{s+1 / 2}\right) \cap L^{2}\left(0, T ; H^{s+1}\right)$ defined by

$$
\mathcal{V}_{M}=\left\{\begin{array}{l}
u \in L^{\infty}\left(0, T ; H^{s+1 / 2}\right) \cap L^{2}\left(0, T ; H^{s+1}\right): \\
\sup _{t \in(0, T)}\|u(t, \cdot)\|_{H^{s+1 / 2}} \leq M, \quad\|u\|_{L^{2}\left(0, T ; H^{s+1}\right)} \leq M
\end{array}\right\} .
$$

For the sake of conciseness, we leave the proof of the following lemma to the reader, since it is very similar to the above a priori estimates.

Lemma 15. Let $\Phi$ be the mapping from $L^{\infty}\left(0, T ; H^{s+1 / 2}\right) \cap L^{2}\left(0, T ; H^{s+1}\right)$ into itself defined by $\Phi(U):=$ $W$, where $W$ is the solution to

$$
\partial_{t} W+2 g \frac{1}{1+R_{0}^{-2|D|}} \mathcal{H} \partial_{\theta} W=f\left(g, \partial_{t} g, \mathbb{Y}, U, \partial_{\theta} U\right) .
$$

There exist $M$ and $T$ small enough such that $\Phi$ is a continuous and contracting mapping from $\mathcal{V}_{M}$ into itself.

Using the above lemmas 121315 leads straightforwardly to the following theorem, thanks to a classical application of the fixed point theorem.

Theorem 16. Let $s \geq 3$. Let $g \in W^{1, \infty}\left(0, T ; H^{s+1 / 2}(\Omega)\right)$, such that

$$
\forall(t, x) \in(0, T) \times \Omega, \quad g(t, x) \geq \alpha>0 .
$$

There exists $M>0$ and $T>0$ small enough such that for $\mathbb{Y}_{0} \in\left(H^{s}(\mathbb{T})\right)^{4}$ and $W_{0} \in H^{s+1 / 2}(\mathbb{T})$ satisfying

$$
\left\|Y_{0}\right\|_{H^{s}} \leq M, \quad\left\|W_{0}\right\|_{H^{s+1 / 2}} \leq M .
$$

Then, the quasilinearized problem (34)-(36) with the initial condition $\left.(\mathbb{Y}, W)\right|_{t=0}=\left(\mathbb{Y}_{0}, W_{0}\right)$ admits a unique solution $(\mathbb{Y}, W)$ such that

$$
\mathbb{Y} \in L^{\infty}\left(0, T ; H^{s}\right), \quad W \in \mathcal{V}_{M}
$$

In order to obtain the well-posedness of the initial free boundary problem, it remains to show that $\left(Y_{1}, Y_{2}\right)$ are effectively the $\left(X_{1}, X_{2}\right)$ solutions to problem 29-30). This is quite obvious since the first two equations of (34) imply that (29) is satisfied. Moreover, at the initial time $W$ satisfies equation (35) and by construction of the equation for $\partial_{t} W$ this equality is propagated at any time. Since we clearly have $W=\partial_{t} Y_{2}, Y_{3}=\partial_{\theta} Y_{1}-Y_{2}$ and $Y_{3}=\partial_{\theta} Y_{2}+Y_{1}$, we infer that $Y_{1}$ and $Y_{2}$ satisfy 29 - (30).

\section{Numerical methods and simulations}

In this section, we describe the first order 2D-Cartesian method used to solve Problem (1) in the Eulerian formalism. This means that the membrane location is detected by the zero of a level-set function $\psi$, which satisfies the transport equation:

$$
\partial_{t} \psi+\mathbf{v} \cdot \nabla \psi=0
$$

where the velocity $\mathbf{v}$ is extended from $\left.\nabla \sigma\right|_{\Gamma_{t}}$ as follows:

$$
\nabla \psi \cdot \nabla \mathbf{v}=0,\left.\quad \mathbf{v}\right|_{\{\psi(t, x)=0\}}=\left.\nabla \sigma\right|_{\{\psi(t, x)=0\}},
$$


instead of equation (1e), all the other equations of (1) remaining unchanged. The numerical computation is based on level set techniques 22] and finite-difference methods on Cartesian grids. The discretized differential operators are derived from centered stencils, on each subdomain delimited by the interface, using the ghost-fluid method [6,9]. The static subproblems $1 \mathrm{ad}-(1 \mathrm{~b})$ and $1 \mathrm{1c}-\mathrm{1 \textrm {d }}$ ) are approximated by methods with first and second order of accuracy, respectively.

The keypoint resides in the superconvergence property of the numerical scheme, which makes it possible to get the solution and its gradient at the same order of accuracy. Hence the protrusion velocity, which is driven by the gradient of the inner solution, is first order accurate, leading to the first order accuracy of the overall method thanks to a well-suited velocity extension. It is presented as follows:

- Poisson-Neumann problem (1a)-1b in the exterior area (ligands),

- Poisson-Dirichlet problem $(1 \mathrm{c})-(1 \mathrm{~d})$ in the interior area (signal),

- Extension of the velocity (42) solve the transport of the level set function 441].

We first give the different numerical schemes used to solve each subproblem, and then we provide some convergence results, with observations about the relevance of Hypothesis (4). Finally, some numerical simulations are presented with a source satisfying Hypothesis (4), that make appear the invadopodia or pseudopodia-like protrusion formations.

Notation 17. The Cartesian grid is the natural choice to avoid remeshing at each time step, as the interface evolves, in particular for parallelization purposes. Throughout the paper, the following notations are used:

- The space steps $\delta x$ and $\delta y$ are denoted by $h$ and such that

$$
h=\delta x=\delta y .
$$

Thus, an accuracy of order $p$ in space must be interpreted as an accuracy at the rate $p$, like $\mathcal{O}\left(h^{p}\right)$.

- The time step is denoted by $\delta t$ and the time discretization is defined by

$$
t^{n}=n \delta t
$$

- The discretized differential operators are denoted by $\Delta^{h}, \nabla^{h}$ and $\partial_{\mathbf{n}^{h}}^{h}$ for the Laplacian operator $\Delta$, the gradient $\nabla$ and the normal derivative $\partial_{\mathbf{n}}$, respectively.

- The grid nodes are denoted by $x_{i, j}$,

- $\varphi_{i, j}^{n}$ denotes the approximation at the point $\left(t^{n}, x_{i, j}\right)$ of any function $\varphi$ defined on $\Omega$,

- $\Omega^{h}$ denotes the set of grid nodes,

- $\mathcal{O}_{t^{n}}^{e, h}$ and $\mathcal{O}_{t^{n}}^{i, h}$ stand for the sets of grid nodes $\left(t^{n}, x_{i, j}\right)$ where $\psi_{i, j}^{n}>0$ and $\psi_{i, j}^{n}<0$, respectively.

$-c^{h}, \sigma^{h}, \psi^{h}$ and $\mathbf{n}^{h}$ are the numerical approximations of $c^{\star}, \sigma, \psi$ and $\mathbf{n}$,

- $\Gamma_{t^{n}}^{h}$ is the set of the intersections of the grid axes and the level 0 of the numerical level set function $\psi^{h}$ at the time $t^{n}$,

- When $j$ (and $n$ ) does not play a role in the numerical stencils, we write $\varphi_{i}$ and $x_{i}$ instead of $\varphi_{i, j}^{n}$ and $x_{i, j}$ to lighten the formulas.

\subsection{Static subproblems}

We first present the numerical methods used to tackle the static problems $11 \mathrm{a})-(1 \mathrm{~b})$ and $(1 \mathrm{c})-(1 \mathrm{~d})$. They are built thanks to the ghost fluid method (see Fedkiw et al. [6] for more details), computing the ghost values with linear extrapolations. 


\subsubsection{Laplace operator discretization}

The Laplace operator is discretized direction by direction. The quantity $u^{h}$ generically denotes $c^{h}$ or $\sigma^{h}$, depending on whether the outer problem or the inner problem is considered. At the regular points, far from the interface, the second order derivatives are discretized with the usual centered stencil (as mentionned before, $j$ is omitted):

$$
\partial_{x x}^{h} u_{i}^{h}=\frac{u_{i+1}^{h}-2 u_{i}^{h}+u_{i-1}^{h}}{h^{2}}, \quad \text { at the regular points. }
$$

Near the interface the centered discretization is not possible since one of the neighbors is on the other side of the interface. At this point, the value is called ghost value and is linearly extrapolated. For instance,

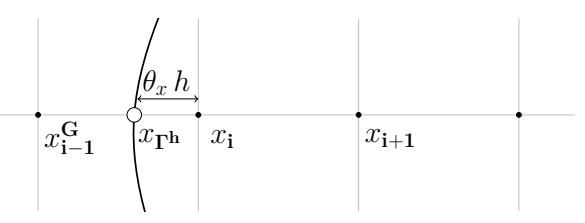

Fig. 7: Ghost-Fluid method for the points near the interface.

the scheme for the second $x$-derivative in figure 7 is given by

$$
\partial_{x x}^{h} u_{i}^{h}=\frac{2}{\theta_{x}\left(1+\theta_{x}\right) h^{2}} u_{\Gamma^{h}}-\frac{2}{\theta_{x} h^{2}} u_{i}^{h}+\frac{2}{\left(1+\theta_{x}\right) h^{2}} u_{i+1}^{h}, \quad \text { near } \Gamma^{h},
$$

where $\theta_{x} h$ denotes the distance of the point $x_{i}$ to the numerical interface $\Gamma^{h}$ in the $x$-axis direction. If the case $\theta_{x}=1$ occurs, we have $u_{\Gamma^{h}}=u_{i-1}^{h}$. The scheme (44) is then equivalent to the standard 3-point stencil scheme (43). Conversely, if $\theta_{x}=0$, the point $x_{i}$ is an interface point and does not belong to the considered domain. It is worth noting that linear extrapolations give a nonconsistent operator near the interface while it is second order accurate at the regular points.

\subsubsection{Exterior static problem (1a)-11b with a Neumann boundary condition.}

The main difficulty lies in the computation of the interface values $c_{\Gamma^{h}}$-denoted by $u_{\Gamma^{h}}$ in 444 - from the Neumann condition. The flux condition at the interface is discretized by evaluating the derivatives of $c^{h}$ on $\Gamma^{h}$, where $\nabla^{h}$ is not defined. We therefore introduce new operators $\partial_{x}^{\Gamma^{h}}$ and $\partial_{y}^{\Gamma^{h}}$, that are directly or indirectly computed from other grid points, depending on whether the interface point belongs to the $x$-axis or to the $y$-axis. The key point lies in the stencil continuity that avoids introducing new unknowns on the interface. In Fig. 8 the point $A$ is on the $y$-axis and the indirect $x$-derivative $\partial_{x}^{\Gamma^{h}} c_{\Gamma^{h}}^{A}$ is computed at the order 1 from the values $c_{\Gamma^{h}}^{A}$ and $c_{1}^{A}$, still using the ghost fluid method and linear extrapolation:

$$
\partial_{x}^{\Gamma^{h}} c_{\Gamma^{h}}^{A}=\frac{c_{1}^{A}-c^{s}}{2 h}=\frac{c_{1}^{A}-c_{\Gamma^{h}}^{A}}{h}
$$

where $c^{s}$ is a ghost value, which is extrapolated at the order 2 from $c_{\Gamma^{h}}^{A}$ and $c_{1}^{A}$. The intermediate value $c_{1}^{A}$ is then interpolated at the order 2 from the known values $c_{i+1, j}$ and $c_{i+1, j+1}$. To ensure the continuity in the stencil arrangements, at the points $A$ and $B$ for instance, the direct $x$-derivative $\partial_{x}^{\Gamma^{h}} c_{\Gamma^{h}}^{B}$ is computed in the same way:

$$
\partial_{x}^{\Gamma^{h}} c_{\Gamma^{h}}^{B}=\frac{c_{1}^{B}-c_{\Gamma^{h}}^{B}}{h}
$$




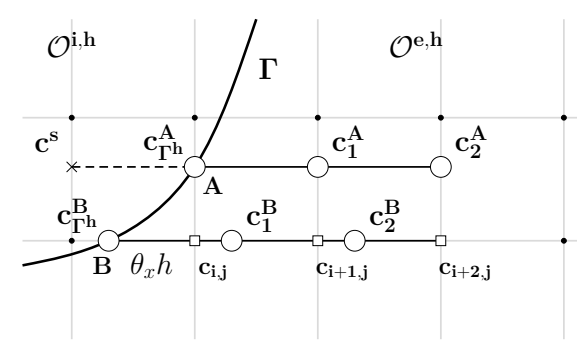

Fig. 8: Continuity of the interface $x$-derivative.

where the intermediate value $c_{1}^{B}$ is interpolated from the known values $c_{i, j}$ and $c_{i+1, j}$, which gives

$$
\partial_{x}^{\Gamma^{h}} c_{\Gamma^{h}}^{B}=\frac{-c_{\Gamma^{h}}^{B}+\theta_{x} c_{i, j}+\left(1-\theta_{x}\right) c_{i+1, j}}{h} .
$$

Thus, if $\theta_{x}$ tends to 0 , both points $x_{\Gamma^{h}}^{A}$ and $x_{\Gamma^{h}}^{B}$ converge towards the point $x_{i, j}$ and both stencils of $\partial_{x}^{\Gamma^{h}} c_{\Gamma^{h}}^{A}$ and $\partial_{x}^{\Gamma^{h}} c_{\Gamma^{h}}^{B}$ tend to

$$
\partial_{x}^{\Gamma^{h}} c_{\Gamma^{h}}=\frac{c_{i+1, j}-c_{i, j}}{h},
$$

which defines the stencil continuity. An example of complete stencil is shown in Fig. 9

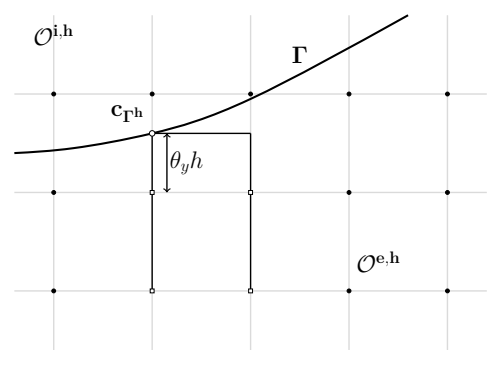

Fig. 9: Complete stencil for the discretization of the Neumann boundary condition.

The flux condition (1b) on the interface is then given by

$$
\partial_{x}^{\Gamma^{h}} c_{\Gamma^{h}} n_{x}^{\Gamma^{h}}+\partial_{y}^{\Gamma^{h}} c_{\Gamma^{h}} n_{y}^{\Gamma^{h}}=-g^{\Gamma^{h}}
$$

where $g^{\Gamma^{h}}$ is known at the order 1 at least. The normal components $n_{x}^{\Gamma^{h}}$ and $n_{y}^{\Gamma^{h}}$ are interpolated at the order 2. Thus, the required value $c_{\Gamma^{h}}$ is obtained from (45) and can be introduced in expression (44) to compute the numerical solution in the exterior domain.

Note that this approach can be extended to a second order accurate method, as proposed by Cisternino and Weynans in [4], by using quadratic extrapolations for both Laplace operator and Neumann condition discretizations. In this case, the Laplace operator discretization is the well-known ShortleyWeller scheme 27] that is consistent of order 1 near the interface. As regards the boundary condition discretization, our continuous approach is different from the approach of Cisternino and Weynans. It avoids considering new interface unknowns and is stable when the interface is very close to a grid point.

Interestingly, the second order method is superconvergent: the gradient of the solution is also second order accurate. Similarly, the first order method used in this study is superconvergent: the gradient of the solution is also first order accurate. 


\subsubsection{Interior static problem with a Dirichlet boundary condition.}

The interface value $\sigma_{\Gamma^{h}}$ is directly obtained from the Dirichlet boundary condition if the data is exactly known on the interface. This case has been studied by Gibou et al. in 9. In particular, the authors numerically highlighted that the use of an inconsistent scheme near the interface does not obstruct the second order accuracy of the method.

In the case of the interior problem, that stands for the signal diffusion, the datum is not exact but implicitly known from the exterior approximated field $c^{h}$. As the exterior field is first order accurate, the second order method for the interior problem also generates a first order solution. However, the computation of $\sigma_{\Gamma^{h}}$ from $c^{h}$ thanks to a linear extrapolation seems to ensure the transmission of the superconvergence property from the outer solution to the inner solution. Hence, the solution and its gradient, the protrusion velocity, are first order accurate, which is the essential aspect to get the overall first order method. This velocity $\mathbf{v}^{h}$ is computed with

$$
\begin{aligned}
& \partial_{x}^{h} u_{i}=\frac{u_{i+1}-u_{i-1}}{2 h}, \quad \text { at the regular points of } \mathcal{O}^{i, h}, \\
& \partial_{x}^{h} u_{i}=\frac{u_{i+1}-u_{i}}{h}, \quad \text { in } \mathcal{O}^{i, h}, \text { near } \Gamma,
\end{aligned}
$$

where (47) is a usual first order discretization of the first derivative at the point near the interface. In coherence with the computation of $\sigma^{h}$, it is built with the ghost fluid method and linear extrapolations of the ghost values.

\subsection{Issues of the dynamics: interface location and velocity extension}

At each numerical time, the coupling of the exterior and the interior problems gives the velocity of the interface. Then, the level set function is advected using the usual forward Euler scheme. The gradient of $\psi$ is discretized with the second order upwind scheme (also called LUD or Beamer-Warming method [30]), which is less dispersive than the first order upwind scheme and therefore ensures a better volume conservation. Then, two main issues arise. As the interface moves, we need first to update at each time step the values of $\theta_{x}$ and $\theta_{y}$, that are used to solve the static problems. Secondly, the velocity, which is defined only in the inner area must be extended to transport the level set function in an appropriate way.

\subsubsection{Computation of the distance to the interface}

Let $x_{i}$ be a grid point near the interface. The quantities $\theta_{x} h$ and $\theta_{y} h$ are the distances of the point $x_{i}$ to the interface $\Gamma^{h}$ in the $x$-axis and $y$-axis directions. Computing these distances with a second order accuracy preserves the first order accuracy of the interface location and the normal vector. This means that $\theta_{x}$ and $\theta_{y}$ should be computed at the order 1 at least. For instance, the computation of $\theta_{x}$ is therefore given by

$$
\begin{array}{ll}
\theta_{x}=\frac{\psi_{i}}{\psi_{i}-\psi_{i-1}}, & \text { if } x_{\Gamma^{h}} \in\left[x_{i-1}, x_{i}\right], \\
\theta_{x}=\frac{-\psi_{i}}{\psi_{i+1}-\psi_{i}}, & \text { if } x_{\Gamma^{h}} \in\left[x_{i}, x_{i+1}\right],
\end{array}
$$

where $\psi_{i}$ and $\psi_{i-1}$ in (48), or $\psi_{i}$ and $\psi_{i+1}$ in 49 are of opposite sign. 


\subsubsection{Velocity extension}

The extension of the velocity is a crucial point of the numerical method. The level set function can be transported without generating discontinuities near the interface only if the velocity field is smoothly defined across the interface and on the whole domain. It therefore has to be extended on each area, from the interface. Several ways of extending the velocity beyond the interface are possible. For instance, we can mention the fast marching method, introduced by Adalsteinsson et al. in [1 or some narrow band approaches with level set reinitialization, as in 17. In this study, we use a PDE method that is consistent with our overall PDE-based approach and avoids the time-consuming process of level set reinitialization at each time step. More precisely, we generate an extended velocity field $\mathbf{w}$ in the whole domain by solving

$$
\begin{array}{ll}
(\nabla \psi \cdot \nabla) \mathbf{w}=0, & \text { on } \Omega, \\
\mathbf{w}=\mathbf{v}, & \text { on } \Gamma,
\end{array}
$$

which means that each component of the extended velocity will be constant along the normal directions. The equation is discretized component by component, introducing the discrete gradient operator $\nabla^{\mathcal{E}}$, which is based on upwind derivatives (from the interface to the rest of the area). For example, to compute the $x$-component of the extended velocity $w_{x}^{h}$ near the interface, as shown in Figure 10 , we solve

$$
\partial_{x}^{\mathcal{E}} w_{x, i}^{h}=\frac{v_{x, \Gamma^{h}}^{h}-w_{x, i}^{h}}{\theta_{x} h}
$$

where $v_{x, \Gamma^{h}}^{h}$ is linearly extrapolated from the values of $v_{x}^{h}$ in $\mathcal{O}^{i, h}$. The classical first order upwind

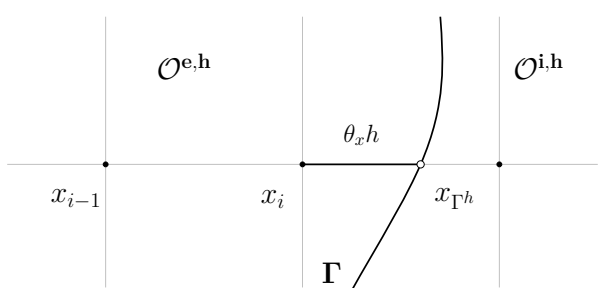

Fig. 10: Example of a forward upwind $x$-derivatives near the interface.

derivatives are used at the other points of $\mathcal{O}^{i, h}$ and $\mathcal{O}^{e, h}$. This method results in a first order extended velocity.

\subsection{Numerical validations}

We simultaneously present two test-cases in order to validate the numerical method and give convergence results. The computational domain is $[-0.5,0.5]^{2}$. The linear systems are inverted thanks to a BiCGStab method. The main difficulty in validating the numerical method lies in the unavailability of any analytical solution, due to the strong non-linearity of the problem. We therefore compare each solution to a reference solution that is performed on a $500 \times 500$ mesh. Errors are computed at the final time $T=1$, when they are supposed to be maximal. Note that errors on $\psi^{h}$ are computed on a tubular area around the interface $\Gamma^{h}$, so that the ridges generated by the velocity extension are avoided and do not disturb the convergence rate computation. For both cases, we define $\psi$ at the initial time as the signed-distance 
function of level 0 the circle of center $(0,0)$ and radius 0.3 . At each numerical time, the boundary data are given by

- test-case 1:

$\forall(r, \theta) \in \Gamma, \quad g_{1}(r, \theta)= \begin{cases}0.05+3 \exp \left(\frac{0.1}{\left(\theta+\frac{10 \pi}{15}\right)\left(\theta+\frac{8 \pi}{15}\right)}\right) & \text { if } \frac{-10 \pi}{15}<\theta<\frac{-8 \pi}{15}, \\ 0.05+2 \exp \left(\frac{0.1}{\left(\theta+\frac{7 \pi}{15}\right)\left(\theta+\frac{5 \pi}{15}\right)}\right) & \text { if } \frac{-7 \pi}{15}<\theta<\frac{-5 \pi}{15}, \\ 0.05 & \text { otherwise }\end{cases}$

- test-case 2:

$\forall(x, y) \in \Gamma, \quad g_{2}(x, y)=0.1[2+\cos (3 \pi(x+y)) \cos (\pi(x+0.3))]$.

At each time, the functions $g_{1}$ and $g_{2}$ satisfy the hypothesis (4), as shown in Figure 11 at the initial time.
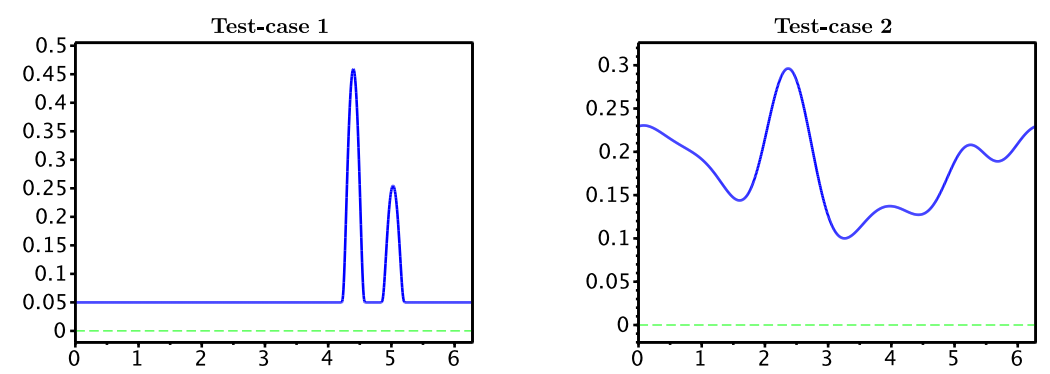

Fig. 11: Functions $g^{0}$ with respect to $\theta$.

Plots of the reference solutions $\sigma^{h}$ at $t=1$ (after 970 time steps for Test-case 1, 601 time steps for Test-case 2) are provided in Fig. 12 and give the general shapes of the cell at the end of each simulation. The convergence results are given on Table 1 and in Figure 13 . For both problems, we observe a first
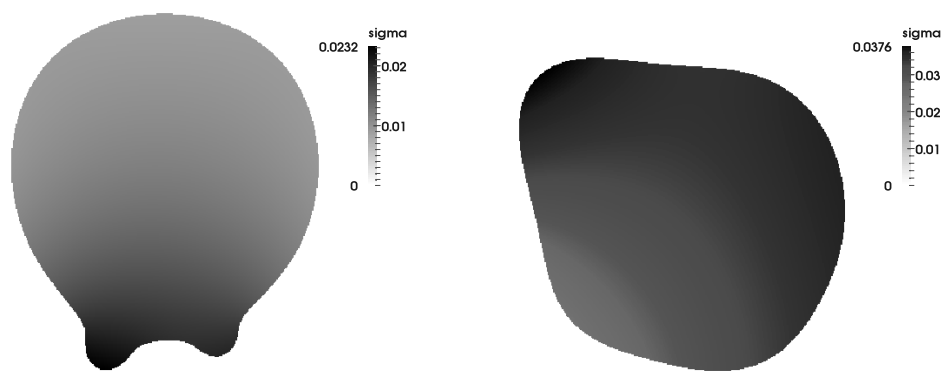

Fig. 12: Plots of $\sigma^{h}$ at $\mathrm{t}=1$ for test-cases 1 (left) and 2 (right).

order numerical convergence for the unknowns $c^{h}, \sigma^{h}$ and $\psi^{h}$, in maximum norm. More surprising, the normal vector $\mathbf{n}^{h}$ is also close to be first order accurate. Again, the overall first order accuracy results from the superconvergence properties and their transmission from the Neumann problem to the Dirichlet problem. They also seem to be preserved by the dynamics. Moreover, the normal vector, which is implied 


\begin{tabular}{|c|c|c|c|c|c|c|c|c|c|}
\hline \multirow[b]{2}{*}{ mesh } & \multirow[b]{2}{*}{$N$} & \multicolumn{2}{|l|}{$c^{h}$} & \multicolumn{2}{|l|}{$\sigma^{h}$} & \multicolumn{2}{|c|}{$\psi^{h}$} & \multicolumn{2}{|l|}{$\mathbf{n}^{h}$} \\
\hline & & error $L^{\infty}$ & order & error $L^{\infty}$ & order & error $L^{\infty}$ & order & error $L^{\infty}$ & order \\
\hline \multicolumn{10}{|c|}{ test-case 1} \\
\hline $32 \times 32$ & 17 & $3.51910^{-3}$ & - & $2.75510^{-3}$ & - & $5.92110^{-3}$ & - & $3.45310^{-1}$ & - \\
\hline $48 \times 48$ & 32 & $2.42410^{-3}$ & 0.92 & $1.60010^{-3}$ & 1.34 & $1.01810^{-2}$ & -1.34 & $2.04210^{-1}$ & 1.30 \\
\hline $72 \times 72$ & 59 & $2.52110^{-3}$ & 0.41 & $1.34810^{-3}$ & 0.88 & $4.39510^{-3}$ & 0.37 & $1.52810^{-1}$ & 1.01 \\
\hline $108 \times 108$ & 105 & $1.92810^{-3}$ & 0.49 & $1.37210^{-3}$ & 0.57 & $3.60810^{-3}$ & 0.40 & $1.14710^{-1}$ & 0.91 \\
\hline $162 \times 162$ & 188 & $1.34310^{-3}$ & 0.59 & $9.78910^{-4}$ & 0.64 & $1.77810^{-3}$ & 0.74 & $7.43110^{-2}$ & 0.95 \\
\hline $243 \times 243$ & 339 & $5.41710^{-4}$ & 0.92 & $3.85110^{-4}$ & 0.97 & $1.14610^{-3}$ & 0.81 & $6.03910^{-2}$ & 0.86 \\
\hline \multicolumn{10}{|c|}{ test-case 2} \\
\hline $32 \times 32$ & 11 & $3.43310^{-3}$ & - & $1.44810^{-3}$ & - & $9.95710^{-3}$ & - & $8.38410^{-2}$ & - \\
\hline $48 \times 48$ & 21 & $2.12910^{-3}$ & 1.18 & $1.04410^{-3}$ & 0.81 & $4.12110^{-3}$ & 2.18 & $4.49210^{-1}$ & 1.54 \\
\hline $72 \times 72$ & 36 & $1.55810^{-3}$ & 0.97 & $6.81410^{-4}$ & 0.93 & $2.83310^{-3}$ & 1.55 & $2.93210^{-2}$ & 1.30 \\
\hline $108 \times 108$ & 64 & $9.64610^{-4}$ & 1.04 & $4.33910^{-4}$ & 0.99 & $2.09310^{-3}$ & 1.28 & $2.68210^{-2}$ & 0.94 \\
\hline $162 \times 162$ & 114 & $5.67910^{-4}$ & 1.11 & $2.67110^{-4}$ & 1.04 & $1.12710^{-3}$ & 1.34 & $1.43010^{-2}$ & 1.09 \\
\hline $243 \times 243$ & 207 & $2.95210^{-4}$ & 1.21 & $1.63610^{-4}$ & 1.08 & $5.97910^{-4}$ & 1.39 & $1.59410^{-2}$ & 0.82 \\
\hline
\end{tabular}

Table 1: Numerical errors and convergence rates for Test-cases 1 and 2.

in the Neumann problem, seems to depend only on the velocity accuracy, which makes it possible for the coupling to be consistent. The details will be further studied in forthcoming work [8].
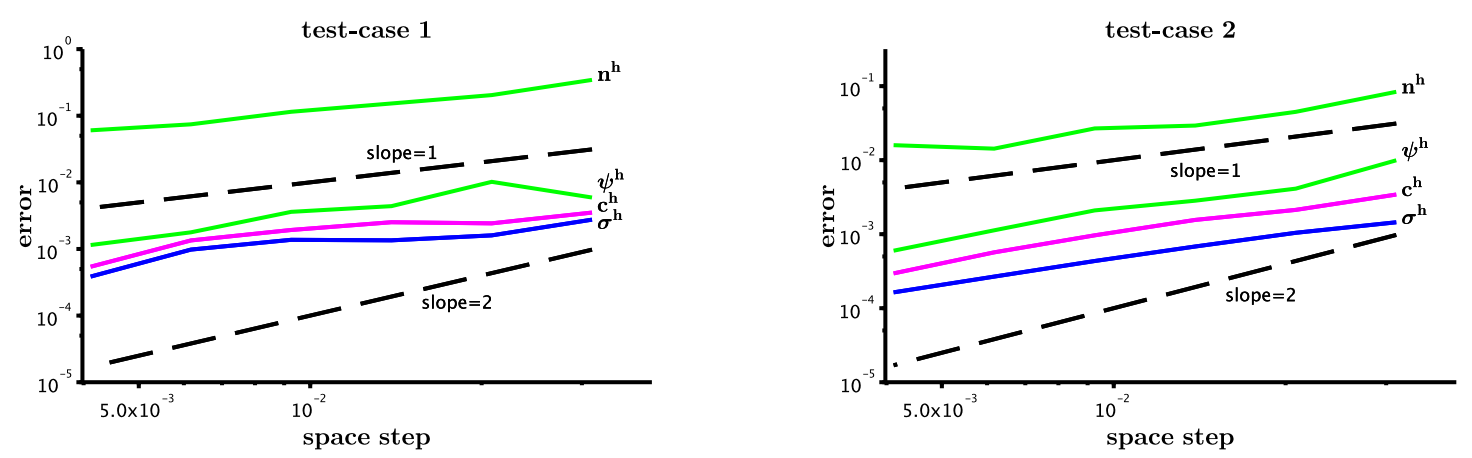

Fig. 13: Convergence curves in $L^{\infty}$-norm.

The divergence-free velocity imposes a theoretical volume conservation, which is preserved accurately by the scheme (see Figure 14), since variations are lower than $0.1 \%$.
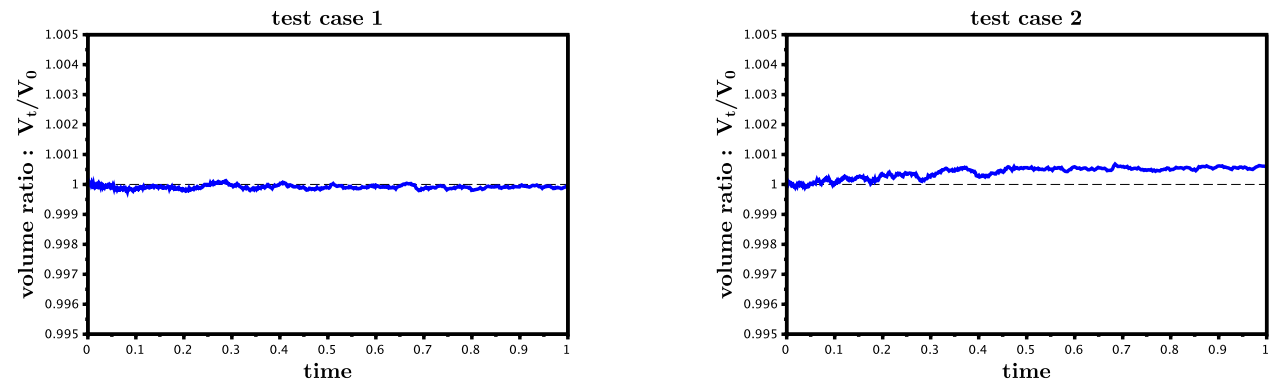

Fig. 14: Volume conservation.

In order to stress Hypothesis (4), we perform the same simulations just by adding a constant $(-0.1)$ 
to the data $g_{1}$ and $g_{2}$ given by (52) and (53). These new data do not verify the positivity condition and instabilities appear on the interface, at some locations where the boundary data is negative or null (Figures 15 16). In particular, Figure 15 shows that, for Test-case $2, g_{2}$ is null at the point $\theta \simeq 3.26$

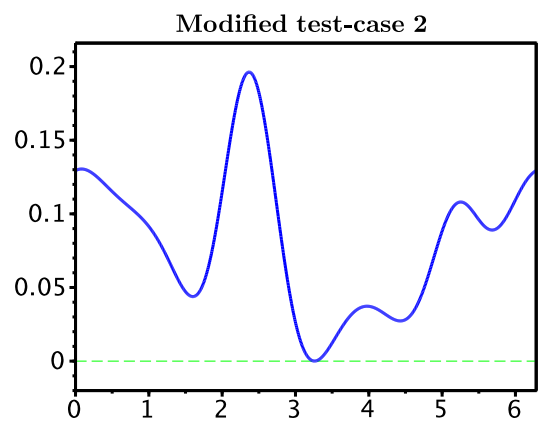

(a) Modified datum $g_{2}$.

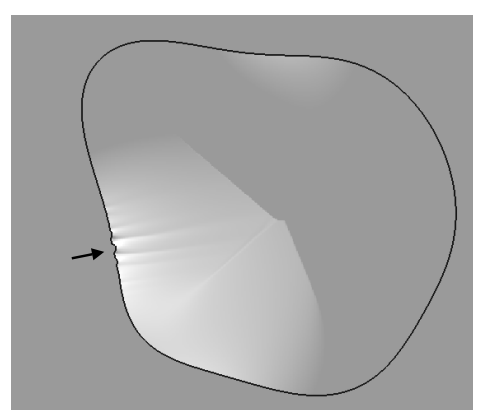

(b) Test-case 2 at $t=1$ time steps).

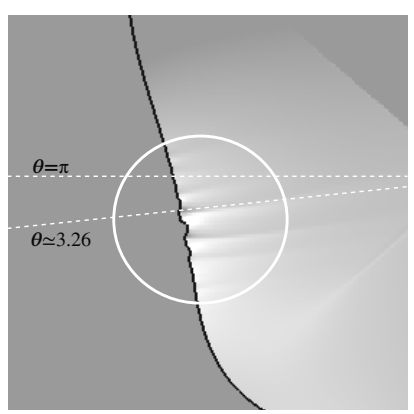

(c) Zoom near the instabilities at $t=1$

Fig. 15: Modified datum $g_{2}$ and numerical instabilities due to the violation of the hypothesis 4. Light areas indicate higher contraction velocities. Black arrows show instabilities. In Fig. 15(c) the zoom shows that instabilities occur in the region where the datum vanishes.

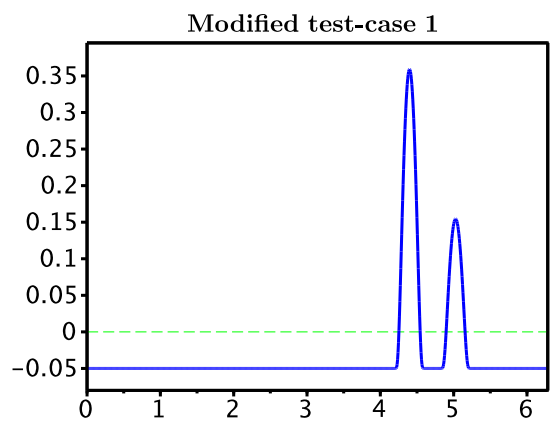

(a) Modified $g_{1}$.

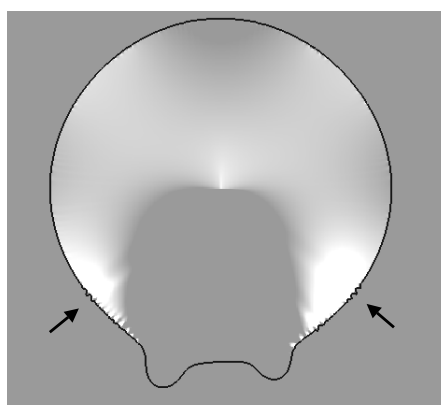

(b) Test-case 1 at $t=0.36$

(114 time steps).

Fig. 16: Modified datum $g_{1}$ and numerical instabilities due to the violation of the hypothesis 4. Light areas indicate higher contraction velocities. Black arrows show instabilities.

and it can be seen in Figure 15(b) that instabilities appear around this position and propagate along the interface. As regards Test-case $1, g_{1}$ does not verify the condition of well-posedness on large areas and the instabilities appear, especially in the regions where the contraction velocity is higher as depicted by the white areas in Fig. 16(b). These observations seem to validate numerically Hypothesis 4 of strict positivity as a necessary condition for well-posedness.

4.4 Biological model behavior: invadopodia and pseudopodia simulations

We present some visualizations of protusion formation, invadopodia and pseudopodial structures, in order to study some behaviors of the model. 


\subsubsection{Problem 1: invadopodia.}

The initial cell is an ellipse of radii 0.4 and 0.15 . The exterior medium is assumed to be homogeneously composed of extracellular matrix, whose concentration value is constant and included with the MMP concentration in the time-dependent function $g$, given as the trace on $\Gamma$ of

$$
\forall(r, \theta) \in \Omega, \quad G(r, \theta)= \begin{cases}0.001+\exp \left(\frac{0.1}{\left(\theta+\frac{9 \pi}{16}\right)\left(\theta+\frac{7 \pi}{16}\right)}\right) \exp (-0.2 t) & \text { if } \frac{-9 \pi}{16}<\theta<\frac{-7 \pi}{16}, \\ 0.001 & \text { otherwise }\end{cases}
$$

The time exponential simulate the decrease in MMP concentration (while the front of the protrusion moves away from the cell nucleus), leading to the expected stabilization of the protrusion. The simulation is performed on a $1000 \times 1000$ mesh until the final time $T=6$, for 476 time steps. We can observe the formation and growth of the invadopodium (Fig. 17(a) 17(d)]. As the MMPs are very localized, the ligands are locally produced and accumulate along the protrusion (Fig. 17(e)) . As expected, they create a gradient of the cytoplasmic signal at the invadopodium (Fig. 17(f)], resulting in its growth. The cell

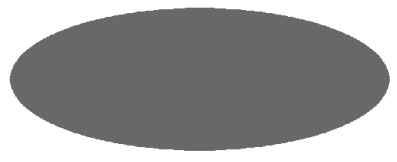

(a) $t=0$.

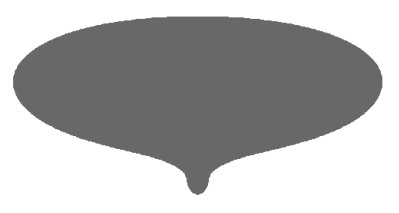

(d) Cell shape at the final time $t=6.0$ (476 time steps).

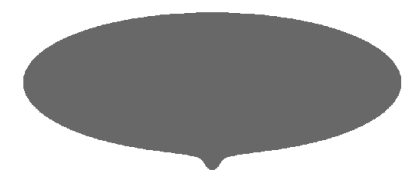

(b) $t=1.0$ (186 time steps).

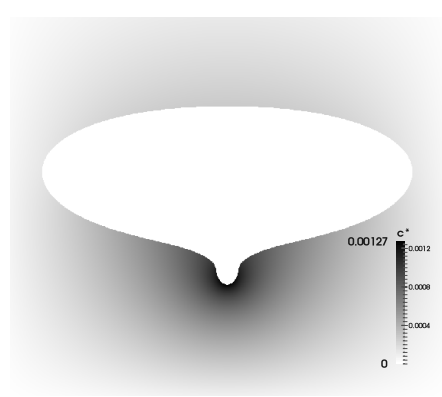

(e) Ligand concentration $\left(c^{h}\right)$ at $t=3.0$.

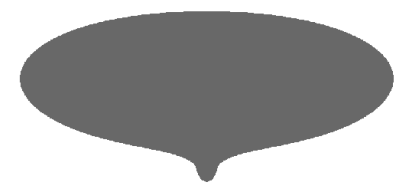

(c) $t=3.2$ (363 time steps).

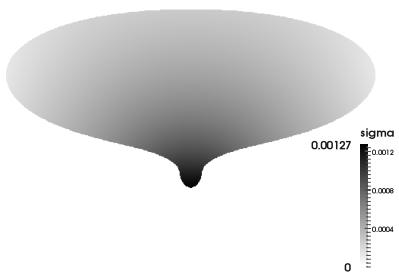

(f) Cytoplasmic signal $\left(\sigma^{h}\right)$ at $t=$ 3.0.

Fig. 17: Simulation of an invadopodium formation.

contracts in the areas that are not protrusive. This observation is supported by the normal velocity values, that show protrusive and contraction areas. This phenomenon can be mathematically explained by the divergence-free velocity, which results in a constant volume of the cell (Fig. 18). As the protrusion occurs only on a small area of the interface, the contraction area is large and the contraction velocities are weak. Consequently, the contraction movement is barely perceptible. Note that, by construction and in addition to the decrease in MMP concentration, the boundary data $g$ behaves as if the area of MMP accumulation expands on the membrane as the protrusion extends. As a result, the protrusion velocity vanishes, indicating the tendency to invadopodium stabilization (Fig. 19). 

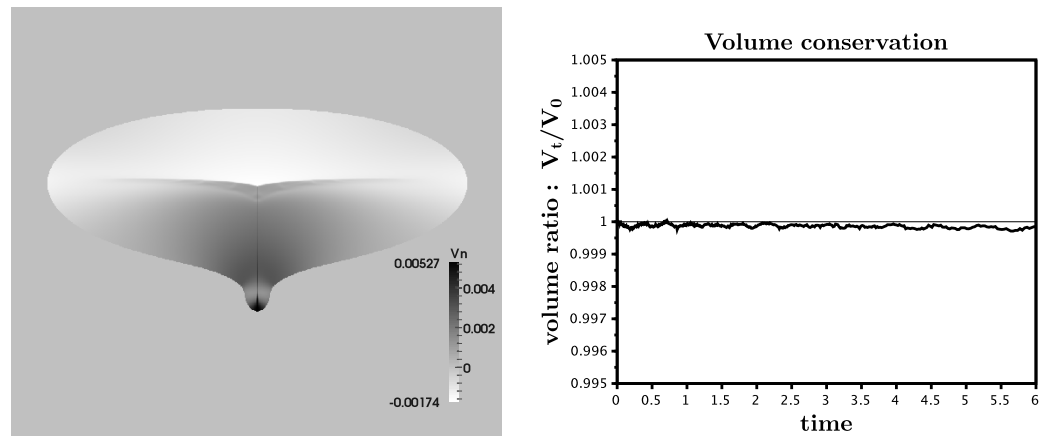

Fig. 18: Normal velocity $(t=6.0)$ and volume conservation with respect to time. Left: the dark areas are for outcoming velocity (protrusion), the light areas are for incoming velocity (contraction). Right: The variations in volume are less than $0.03 \%$.
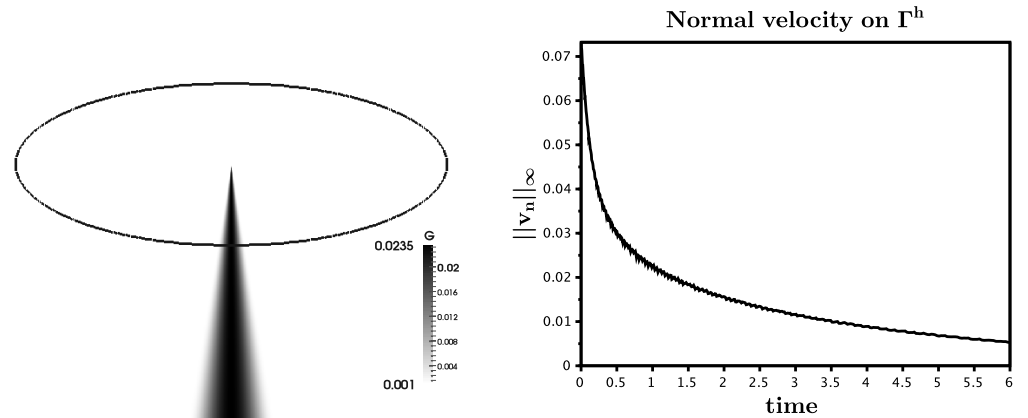

Fig. 19: Stabilization of the invadopodium (right), related to the time-dependent boundary data, given at each time by the trace of $G$ (left).

\subsubsection{Problem 2: pseudopodia-like projection.}

For the pseudopodia simulation, we use the model including modifications given by (2). The initial cell is an ellipse of radii 0.24 and 0.14 , and center $(-0.15,0)$. The simulation is performed with the data $g$ given by

$$
\begin{aligned}
& g(-0.5, y)=0.1, \quad g(0.5, y)=0.4 \quad \forall y \in[-0.5,0.5], \\
& g(x,-0.5)=g(x, 0.5)=0.25+0.3 x, \quad \forall x \in[-0.5,0.5] .
\end{aligned}
$$

The localization function $\kappa$ is given at each time $t^{n}$ as a function of $\sigma$ :

$$
\kappa^{n}=0.001+0.05\left(1+\tanh \left(\lambda\left(\sigma^{n}-\bar{\sigma}^{n}\right)\right)\right) .
$$

The signal $\sigma$ gives the polarization of the cell, generated by the external gradient, and the direction of the protrusion. However the velocity can exist only in the area where the proteins required for the actin polymerization are located, which is described by (56). The threshold $\bar{\sigma}^{n}$ delimits the areas with and without proteins. As these areas evolve as the protrusion grows, we define $\bar{\sigma}$ as a time-dependent linear interpolation of the extrema of $\sigma$ :

$$
\bar{\sigma}^{n}=\left(0.2-0.03 t^{n}\right) \sigma_{\min }+\left(0.8+0.03 t^{n}\right) \sigma_{\max } .
$$

The slope of the curve at the limit of both areas is given by the parameter $\lambda$ also defined from the extrema of $\sigma$ :

$$
\lambda=\frac{10}{\sigma_{\max }-\sigma_{\min }} .
$$


The simulation is performed on a $500 \times 500$ grid until the final time $T=5$, for 320 time steps. We provide plots of the cell shape during the simulation in Figure 20. The blood vessel is on the right edge of the

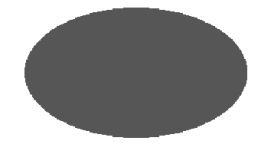

(a) $t=0$.

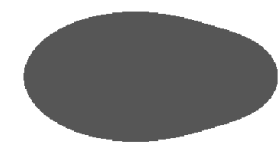

(b) $t=1.6$ (117 time steps).

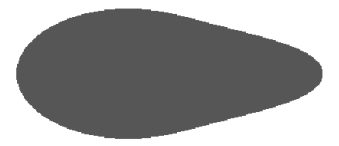

(c) $t=5$ (320 time steps).

Fig. 20: Simuation of pseudopodia formation.

computational domain. The gradient of chemoattractant polarizes the cell from the left to the right, that leads to the formation of the protrusion on the right of the cell and to its growth towards the blood vessel. The pseudopodium width depends only on the definition of the localization function $\kappa$ and more precisely on the threshold $\bar{\sigma}$. Since the velocity is not divergence-free, the pseudopodium growth results in an increased volume (Fig. 21(a) . As the cell elongates on a wide leading front, the distribution of the signal spreads out and the normal velocity decreases (Fig. 21(b)].

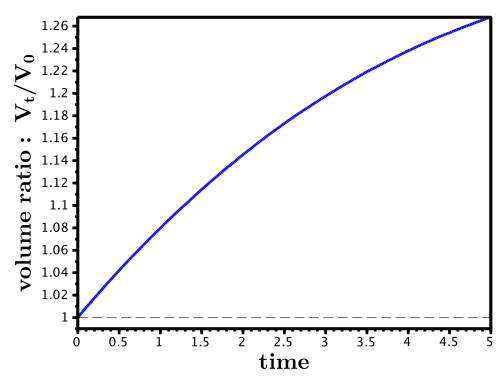

(a) Increase in volume.

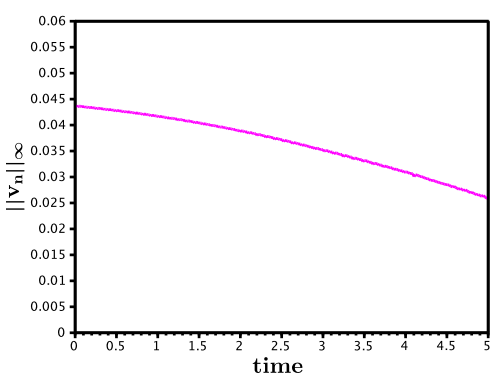

(b) Decrease in normal velocity.

Fig. 21: Behavior of the model for pseudopodia formation.

\section{Discussion and perspectives}

Our core model for protrusion formation under external gradient, takes the cytoplasmic membrane into account as a free boundary. It has been proved that the free boundary problem is well-posed under the sign condition (4) that has been numerically validated. The consistency of the numerical method is based on superconvergence properties of the scheme that are not all well understood and that will be specifically studied in a future work [8]. Roughly speaking the gradient of the numerical solutions to the static subdomains problems are at the same order of the solutions themselves, which prevents loss of accuracy. 
Numerical simulations provide protrusion-like shapes, validating the derivation of the model. We emphasize that these are the first steps towards a precise description of cell protrusion formation. In the case of invadopodia, cytoplasmic and membrane dynamics of MMPs remain to be explored to obtain a realistic time-dependent function $g$, in the purpose, for instance, to calibrate the model or study the stabilization of invadopodia, as in [25. The issue of the divergence-free velocity also arises: does the cell deform at constant volume or is the membrane extended? Regarding pseudopodia, it seems easier to get a realistic boundary data since it just corresponds to the concentration of chemoattractant released by the neighboring blood vessel. Moreover, numerical simulations are more straightforward because larger protrusions require less grid refinement and generate less numerical instabilities. However, the issue of the cell polarization and the cytoplasmic protein distribution, that is roughly approximated by the function $\kappa$ in our study, also remains to deepen.

Note that in order to achieve a complete migratory behavior, it should be necessary to introduce many other biological processes, especially cell-collagen adhesions and myosin-induced contractility. Then we could obtain more realistic simulations by combining pseudopodia and invadopodia for a cell that would migrate in a matrix of collagen fibers. In conclusion, we have proposed and studied theoretically and numerically a core model that is a new step in the modeling of protrusion formation at the cellular level. This topic is the cornerstone of the cell migration understanding, especially as regards the cancer cell invasion and migration. However, many biological issues, such as MMPs and cell polarization dynamics or velocity definition, and mathematical issues, like 3D-analysis and numerical analysis, remain to be further studied.

Acknowledgements This study has been carried out with financial support from the JSPS Core to Core program Advanced Research Networks.

O.G and C.P has been partly supported by the French National Research Agency (ANR) in the frame of the "Investments for the future" Programme IdEx Bordeaux - CPU (ANR-10-IDEX-03-02).

Numerical simulations presented in this paper were carried out using the PLAFRIM experimental testbed, being developed under the Inria PlaFRIM development action with support from LABRI and IMB and other entities: Conseil Régional d'Aquitaine, FeDER, Université de Bordeaux and CNRS (see https://plafrim.bordeaux.inria.fr/).

The authors would like to thank very warmly Professor Thierry Colin for his advices and suggestions that were helpful in the analytical and numerical study of the cell migration problem. They are also grateful to the reviewers that help in the improvement of the present paper.

\section{A The quasistatic 2-phase Hele-Shaw (Muskat) problem}

In this Appendix we consider the quasistatic 2-phase Hele-Shaw also called Muskat problem set in the domain $\Omega$ defined as in Fig. 4(b) We use the same notation as before for the geometry. Let $g$ be a given function defined in $\mathbb{R}^{+} \times \mathbb{R}^{2}$. The PDE system satisfied by $\gamma, c^{\star}$, and $\sigma$ reads

$$
\left.\gamma\right|_{t=0}=\gamma_{0}, \quad \text { and } \quad \partial_{t} \gamma=\nabla\left(\sigma-c^{\star}\right)(\gamma(t, \theta)), \quad \forall t>0, \quad \forall \theta \in \mathbb{R} / 2 \pi \mathbb{Z},
$$

where $\forall t \geq 0$, one has

$$
\begin{aligned}
& \Delta c^{\star}=0, \quad x \in \mathcal{O}_{t}^{e}, \\
& \left.c^{\star}\right|_{\partial \Omega}=0,\left.\quad c^{\star}\right|_{\Gamma_{t}}=\left.g\right|_{\Gamma_{t}}, \\
& \Delta \sigma=0, \quad t \in[0, T], \quad x \in \mathcal{O}_{t}^{i}, \\
& \left.\sigma\right|_{\Gamma_{t}}=\left.c^{\star}\right|_{\Gamma_{t}} .
\end{aligned}
$$

From the numerical point of view, since Dirichlet conditions are imposed on both sides of $\Gamma_{t}$, our numerical schemes can be easily used with the same order of accuracy. In the following we show how to obtain the well-posedness result:

Theorem 18 (Well-posedness of the quasistatic 2-phase Stefan problem). Let $\Omega$ be a smooth domain of $\mathbb{R}^{2}$ which strictly contains the unit disk, and denote by $\Gamma_{0}$ the initial location of the interface, given as a perturbation of the unit circle : $\Gamma_{0}=\left\{e^{i \theta}+\xi_{0}, \theta \in \mathbb{T}\right\}$. 
Let $s \geq 3$, and let $g \in W^{1, \infty}\left(\mathbb{R}^{+} ; H^{s+3 / 2}(\Omega)\right)$ such that for a given $\alpha>0$, and $M>0$,

$$
-\nabla g \cdot \partial_{\theta} \zeta_{0}^{\perp}-\frac{1+R_{0}^{-2|D|}}{2 R_{0} \log \left(R_{0}\right)} \mathcal{P}_{0}(g) \geq \alpha>0, \quad\|g\|_{W^{1, \infty}\left(\mathbb{R}_{;}+H^{s+3 / 2}\right)} \leq M, \quad \text { with } \zeta_{0}(\theta)=e^{i \theta}
$$

There exist $\delta>0$ and $T>0$ small enough such that if

$$
\left\|\xi_{0}\right\|_{H^{s}(\mathbb{T})} \leq \delta
$$

then, there exists a unique solution $\left(\gamma, c^{\star}, \sigma\right)$ on $(0, T)$ to problem s7 such that

$$
\gamma \in L^{\infty}\left(0, T ; H^{s}(\mathbb{T})\right) \cap L^{2}\left(0, T ; H^{s+1}\right),
$$

and for almost any $t \in(0, T)$,

$$
c^{\star} \in H^{s+1 / 2}\left(\mathcal{O}_{t}^{e}\right), \quad \sigma \in H^{s+1 / 2}\left(\mathcal{O}_{t}^{i}\right)
$$

A.1 Equivalent problem on the torus $\mathbb{T}=\mathbb{R} / 2 \pi \mathbb{Z}$

We write an equivalent problem set on the torus $\mathbb{T}=\mathbb{R} / 2 \pi \mathbb{Z}$. Deriving with respect to $\theta$ the Dirichlet traces of $\sigma$ and $c^{\star}$ on $\Gamma_{t}$, we get:

$$
\partial_{\theta}\left(\left(\sigma-c^{\star}\right)(\gamma)\right)=0=\partial_{t} \gamma \cdot \partial_{\theta} \gamma
$$

Then, setting as before

$$
X_{1}=\xi \cdot \partial_{\theta} \zeta_{0}^{\perp}, \quad X_{2}=\xi \cdot \partial_{\theta} \zeta_{0},
$$

we get the first equation which corresponds to 29 for the cell migration problem:

$$
\left(1+X_{1}+\partial_{\theta} X_{2}\right) \partial_{t} X_{2}=-\left(\partial_{\theta} X_{1}-X_{2}\right) \partial_{t} X_{1}
$$

Then, thanks to Lemma 6, we get:

$$
\begin{aligned}
\nabla c^{\star} \cdot \partial_{\theta} \zeta_{0} & =\mathcal{L}_{\mathrm{e}}\left(\nabla c^{\star} \cdot \partial_{\theta} \zeta_{0}^{\perp}\right) \\
& =\mathcal{L}_{\mathrm{e}}\left(\nabla \sigma \cdot \partial_{\theta} \zeta_{0}^{\perp}\right)-\mathcal{L}_{\mathrm{e}}\left(\partial_{t} \xi \cdot \partial_{\theta} \zeta_{0}^{\perp}\right) \\
& =\mathcal{L}_{\mathrm{e}} \mathcal{L}_{\mathrm{i}}\left(\nabla \sigma \cdot \partial_{\theta} \zeta_{0}\right)-\mathcal{L}_{\mathrm{e}}\left(\partial_{t} \xi \cdot \partial_{\theta} \zeta_{0}^{\perp}\right) \\
& =\mathcal{L}_{\mathrm{e}} \mathcal{L}_{\mathrm{i}}\left(\nabla c^{\star} \cdot \partial_{\theta} \zeta_{0}\right)+\mathcal{L}_{\mathrm{e}} \mathcal{L}_{\mathrm{i}}\left(\partial_{t} \xi \cdot \partial_{\theta} \zeta_{0}\right)-\mathcal{L}_{\mathrm{e}}\left(\partial_{t} \xi \cdot \partial_{\theta} \zeta_{0}^{\perp}\right)
\end{aligned}
$$

Now observe that around $\xi=0$, the operator writes (see Lemma 6]:

$$
\mathcal{L}_{\mathrm{e}} \mathcal{L}_{\mathrm{i}} \sim \frac{1-R_{0}^{-2|D|}}{1+R_{0}^{-2|D|}} \mathcal{H}^{2}=\mathcal{P}_{0}-\frac{1-R_{0}^{-2|D|}}{1+R_{0}^{-2|D|}}
$$

thus for $\xi$ small enough,

$$
\left(1-\mathcal{L}_{\mathrm{e}} \mathcal{L}_{\mathrm{i}}\right) \sim \frac{2}{1+R_{0}^{-2|D|}}-\mathcal{P}_{0},
$$

which is invertible. Therefore $\left(1-\mathcal{L}_{\mathrm{e}} \mathcal{L}_{\mathrm{i}}\right)$ is invertible for $\xi$ small enough and its inverse operator $\mathcal{A}$ reads

$$
\mathcal{A}=\left(1-\mathcal{L}_{\mathrm{e}} \mathcal{L}_{\mathrm{i}}\right)^{-1} \sim \frac{1+R_{0}^{-2|D|}}{2}\left(1+\frac{1+R_{0}^{-2|D|}}{1-R_{0}^{-2|D|}} \mathcal{P}_{0}\right)
$$

and we get

$$
\nabla c^{\star} \cdot \partial_{\theta} \zeta_{0}=\mathcal{A} \mathcal{L}_{\mathrm{e}}\left(-\partial_{t} X_{1}+\mathcal{L}_{\mathrm{i}}\left(\partial_{t} X_{2}\right)\right)
$$

Similarly, we obtain

$$
\begin{aligned}
\nabla c^{\star} \cdot \partial_{\theta} \zeta_{0}^{\perp} & =-\partial_{t} \xi \cdot \partial_{\theta} \zeta_{0}^{\perp}+\nabla \sigma \cdot \partial_{\theta} \zeta_{0}^{\perp} \\
& =-\partial_{t} \xi \cdot \partial_{\theta} \zeta_{0}^{\perp}+\mathcal{L}_{\mathrm{i}}\left(\nabla \sigma \cdot \partial_{\theta} \zeta_{0}\right) \\
& =-\partial_{t} X_{1}+\mathcal{L}_{\mathrm{i}}\left(\partial_{t} X_{2}\right)+\mathcal{L}_{\mathrm{i}} \mathcal{L}_{\mathrm{e}}\left(\nabla c^{\star} \cdot \partial_{\theta} \zeta_{0}^{\perp}\right)
\end{aligned}
$$

and thus

$$
\nabla c^{\star} \cdot \partial_{\theta} \zeta_{0}^{\perp}=\mathcal{B}\left(-\partial_{t} X_{1}+\mathcal{L}_{\mathrm{i}}\left(\partial_{t} X_{2}\right)\right),
$$


where similarly

$$
\mathcal{B}=\left(1-\mathcal{L}_{\mathrm{i}} \mathcal{L}_{\mathrm{e}}\right)^{-1} \sim \frac{1+R_{0}^{-2|D|}}{2}\left(1+\frac{1+R_{0}^{-2|D|}}{1-R_{0}^{-2|D|}} \mathcal{P}_{0}\right)
$$

We are now ready to obtain the second equation, which corresponds to equation 30. Since

$$
\begin{aligned}
\nabla g \cdot \partial_{\theta} \gamma & =\nabla c^{\star} \cdot \partial_{\theta} \zeta_{0}+\nabla c^{\star} \cdot \partial_{\theta} \xi \\
& =\left(1+\partial_{\theta} \xi \cdot \partial_{\theta} \zeta_{0}\right) \nabla c^{\star} \cdot \partial_{\theta} \zeta_{0}+\partial_{\theta} \xi \cdot \partial_{\theta} \zeta_{0}^{\perp}\left(\nabla c^{\star} \cdot \partial_{\theta} \zeta_{0}^{\perp}\right), \\
& =\left\{\left(1+X_{1}+\partial_{\theta} X_{2}\right) \mathcal{A} \mathcal{L}_{\mathrm{e}}+\left(\partial_{\theta} X_{1}-X_{2}\right) \mathcal{B}\right\}\left(-\partial_{t} X_{1}+\mathcal{L}_{\mathrm{i}}\left(\partial_{t} X_{2}\right)\right),
\end{aligned}
$$

hence we infer

$$
\begin{aligned}
& \left\{\left(1+X_{1}+\partial_{\theta} X_{2}\right) \mathcal{A} \mathcal{L}_{\mathrm{e}}+\left(\partial_{\theta} X_{1}-X_{2}\right) \mathcal{B}\right\}\left(-\partial_{t} X_{1}+\mathcal{L}_{\mathrm{i}}\left(\partial_{t} X_{2}\right)\right) \\
& =\left(1+X_{1}+\partial_{\theta} X_{2}\right) \nabla g \cdot \partial_{\theta} \zeta_{0}+\left(\partial_{\theta} X_{1}-X_{2}\right) \nabla g \cdot \partial_{\theta} \zeta_{0}^{\perp} .
\end{aligned}
$$

In addition to the results of Lemma 6] it is necessary to use the following equality:

$$
\left(\mathcal{P}_{0}+\mathcal{A}_{3}(\xi)\right)\left(\nabla c^{\star} \cdot \partial_{\theta} \zeta_{0}^{\perp}\right)=-\frac{1}{R_{0} \log \left(R_{0}\right)} \mathcal{P}_{0}(g),
$$

which can be easily verified by Fourier calculus. Thanks to the avove equality, we infer that

$$
\mathcal{P}_{0}\left(\partial_{t} X_{1}\right)+\mathcal{A}_{4}(\xi)\left(\partial_{t} X_{2}\right)+\mathcal{A}_{5}(\xi) \partial_{t} X_{1}=\frac{1}{R_{0} \log \left(R_{0}\right)} \mathcal{P}_{0}(g),
$$

where the operators $\mathcal{A}_{j}(\xi), j=3,4,5$ belong to $L_{0}(2, s ; 2)$ similarly to the operators $\left(\mathcal{A}_{i}(\xi)\right)_{i=1,2}$ of Lemma 6

\section{A.2 Quasilinearization}

Now let $\left(Y_{i}\right)_{i=1, \cdots, 4}$ and $W$ be defined as

$$
\begin{aligned}
& Y_{1}=X_{1}, \quad Y_{2}=X_{2}, \\
& Y_{3}=\partial_{\theta} X_{1}-X_{2}, \quad Y_{4}=\partial_{\theta} X_{2}+X_{1}, \\
& W=\partial_{t} X_{1} .
\end{aligned}
$$

Equations 59-62 write now:

$$
\begin{aligned}
& \partial_{t} Y_{1}=W, \\
& \partial_{t} Y_{2}=-\frac{Y_{3}}{1+Y_{4}} W, \\
& \partial_{t} Y_{3}=\partial_{\theta} W, \\
& \partial_{t} Y_{4}=\partial_{\theta}\left(-\frac{Y_{3}}{1+Y_{4}} W\right),
\end{aligned}
$$

and on $W$ we get

$$
\left\{\left(1+Y_{4}\right) \mathcal{A} \mathcal{L}_{\mathrm{e}}+Y_{3} \mathcal{B}\right\} W-\left\{\left(1+Y_{4}\right) \mathcal{A} \mathcal{L}_{\mathrm{e}}+Y_{3} \mathcal{B}\right\} \mathcal{L}_{\mathrm{i}}\left(\frac{Y_{3}}{1+Y_{4}} W\right)=\left(1+Y_{4}\right) \nabla g \cdot \partial_{\theta} \zeta_{0}+Y_{3} \nabla g \cdot \partial_{\theta} \zeta_{0}^{\perp}
$$

Deriving equation 64 with respect to time leads to the following non-local PDE for $W$ around $\mathbb{Y} \sim 0$ :

$$
\frac{1-R_{0}^{-2|D|}}{2} \mathcal{H} \partial_{t} W+\frac{1+R_{0}^{-2|D|}}{2} W \partial_{\theta} W+\left(\nabla g \cdot \partial_{\theta} \zeta_{0}^{\perp}\right) \partial_{\theta} W=\text { source term, }
$$

or equivalently, setting $\tilde{W}=\mathcal{H} W$ :

$$
\frac{1+R_{0}^{-2|D|}}{2} \partial_{t} \tilde{W}-\left(\nabla g \cdot \partial_{\theta} \zeta_{0}^{\perp}\right) \mathcal{H} \partial_{\theta} \tilde{W}-\frac{1+R_{0}^{-2|D|}}{2} W \mathcal{H} \partial_{\theta} \tilde{W}=\text { source term }
$$

Using equality 63, observe that

$$
W=\mathcal{P}_{0}(W)-\mathcal{H}(\tilde{W})=\frac{1}{R_{0} \log \left(R_{0}\right)} \mathcal{P}_{0}(g)-\mathcal{H}(\tilde{W}),
$$


hence $\tilde{W}$ satisfies

$$
\frac{1+R_{0}^{-2|D|}}{2} \partial_{t} \tilde{W}-\left[\left(\nabla g \cdot \partial_{\theta} \zeta_{0}^{\perp}\right)+\frac{1+R_{0}^{-2|D|}}{2 R_{0} \log \left(R_{0}\right)} \mathcal{P}_{0}(g)\right] \mathcal{H} \partial_{\theta} \tilde{W}+\frac{1}{2} \partial_{\theta}\left((\mathcal{H} \tilde{W})^{2}\right)=\text { source term }
$$

from which we get the well-posedness in $L^{\infty}\left(0, T ; H^{s}\right) \cap L^{2}\left(0, T ; H^{s+1}\right)$ under the sign condition

$$
-\nabla g \cdot \partial_{\theta} \zeta_{0}^{\perp}-\frac{1+R_{0}^{-2|D|}}{2 R_{0} \log \left(R_{0}\right)} \mathcal{P}_{0}(g) \geq \alpha>0,
$$

with $g \in W^{1, \infty}\left(0, T ; H^{s+3 / 2}\right)$.

\section{References}

1. D. Adalsteinsson and J.A Sethian. The Fast Construction of Extension Velocities in Level Set Methods. Journal of Computational Physics, 148(1):2-22, 1999.

2. N. R. Alexander, K.M. Branch, A. Parekh, E.S. Clark, I.C. Iwueke, S.A. Guelcher, and A.M. Weaver. Extracellular matrix rigidity promotes invadopodia activity. Current Biology, 18(17):1295 - 1299, 2008.

3. K. M. Branch, D. Hoshino, and A. M. Weaver. Adhesion rings surround invadopodia and promote maturation. Biology Open, page BIO20121867, June 2012.

4. M. Cisternino and L. Weynans. A parallel second order cartesian method for elliptic interface problems. Communications in Computational Physics, 12:1562-1587, June 2012.

5. H. Enderling, N. Alexander, E.S. Clark, K.M. Branch, L. Estrada, C. Crooke, J. Jourquin, N. Lobdell, M.H. Zaman, S.A. Guelcher, A.R.A. Anderson, and A. Weaver. Dependence of invadopodia function on collagen fiber spacing and cross-linking: Computational modeling and experimental evidence. Biophysical Journal, 95(5):2203-2218, 2008.

6. R. P. Fedkiw, T. Aslam, B. Merriman, and S. Osher. A Non-oscillatory Eulerian Approach to Interfaces in Multimaterial Flows (the Ghost Fluid Method). Journal of Computational Physics, 152(2):457-492, 1999.

7. P. Friedl and K. Wolf. Tumour-cell invasion and migration: diversity and escape mechanisms. Nat Rev Cancer, 3(5):362-374, 052003.

8. O. Gallinato and C. Poignard. Superconvergent second order Cartesian method for a free boundary model of invadopodia. Submitted, 2016.

9. F. Gibou, R.P. Fedkiw, L.T. Cheng, and M. Kang. A Second-Order-Accurate Symmetric Discretization of the Poisson Equation on Irregular Domains. Journal of Computational Physics, 176:205-227, 2002.

10. M. Herant and M. Dembo. Form and function in cell motility: From fibroblasts to keratocytes. Biophysical Journal, 98(8):1408 - 1417, 2010.

11. W. R. Holmes and L. Edelstein-Keshet. A comparison of computational models for eukaryotic cell shape and motility. PLoS Comput Biol, 8(12):e1002793, 2012.

12. D. Hoshino, N. Koshikawa, T. Suzuki, V. Quaranta, A. M. Weaver, M. Seiki, and K. Ichikawa. Establishment and validation of computational model for mt1-mmp dependent ecm degradation and intervention strategies. PLoS Comput Biol, 8(4):1-10, 042012.

13. T. Iguchi. On the irrotational flow of incompressible ideal fluid in a circular domain with free surface. Publ. Res. Inst. Math. Sci., 34(6):525-565, 1998.

14. T. Kato. Nonlinear semigroups and evolution equations. J. Math. Soc. Japan, 19:508-520, 1967.

15. D. Lannes. Well-posedness of the water-waves equations. J. Amer. Math. Soc., 18(3):605-654 (electronic), 2005.

16. H. Levine and W.-J. Rappel. The physics of eukaryotic chemotaxis. Phys Today, 66(2), 2013.

17. P. Macklin and J. Lowengrub. Evolving interfaces via gradients of geometry-dependent interior Poisson problems: application to tumor growth. Journal of Computational Physics, 203(1):191-220, 2005.

18. A. F. M. Marée, V. A. Grieneisen, and L. Edelstein-Keshet. How cells integrate complex stimuli: The effect of feedback from phosphoinositides and cell shape on cell polarization and motility. PLoS Comput Biol, 8(3):1-20, 032012.

19. A. F. M. Marée, A. Jilkine, A. Dawes, V. A. Grieneisen, and L. Edelstein-Keshet. Polarization and movement of keratocytes: A multiscale modelling approach. Bulletin of Mathematical Biology, 68(5):1169-1211, 2006.

20. A. Mogilner. On the edge: modeling protrusion. Current Opinion in Cell Biology, 18(1):32-39, 2006. Cell structure and dynamics.

21. V. I. Nalimov. The Cauchy-Poisson problem. Dinamika Splošn. Sredy, Vyp. 18 Dinamika Zidkost. so Svobod. Granicami:104-210, 254, 1974.

22. S. Osher and J.A. Sethian. Fronts Propagating with Curvature Dependent Speed: Algorithms Based on HamiltonJacobi Formulations. Journal of Computational Physics, 79(1):12-49, 1988.

23. A. Pathak and S. Kumar. Biophysical regulation of tumor cell invasion: moving beyond matrix stiffness. Integr. Biol., 3:267-278, 2011. 
24. A. J. Ridley, M. A. Schwartz, K. Burridge, R. A. Firtel, M. H. Ginsberg, G. Borisy, J. T. Parsons, and A. R. Horwitz. Cell Migration: Integrating Signals from Front to Back. Science, 302(5651):1704-1709, 2003.

25. T. Saitou, M. Rouzimaimaiti, N. Koshikawa, M. Seiki, K. Ichikawa, and T. Suzuki. Mathematical modeling of invadopodia formation. J Theor Biol., 298, pages 138-146, 2012.

26. P.M. Schoumacher, R.D. Goldman, D. Louvard, and D.M. Vignjevic. Actin,microtubules, and vimentin intermediate filament cooperate for elongation of invadopodia. J Cell Biol., 189(3):541-556, 2010.

27. G. H. Shortley and R. Weller. The Numerical Solution of Laplace's Equation. Journal of Applied Physics, 9(5):334, 1938.

28. C. Stock and A. Schwab. Role of the Na+/H+ exchanger NHE1 in cell migration. Acta Physiologica, 187(1-2):149-157, 2006.

29. D. Strychalski, W. an Adalsteinsson and T.C. Elston. Simulating biochemical signaling networks in complex moving geometries. SIAM Journal on Scientific Computing, 32(5):3039-3070, 2010.

30. R. F. Warming and Richard M. Beam. Upwind second-order difference schemes and applications in aerodynamic flows. AIAA Journal, 14(9):1241-1249, 2016/07/06 1976.

31. C. Wolgemuth, J. Stajic, and A. Mogilner. Redundant mechanisms for stable cell locomotion revealed by minimal models. Biophysical Journal, 101(3):545-553, 2011.

32. M. Yang, D. J. Kozminski, L. A. Wold, R. Modak, J. D. Calhoun, L. L. Isom, and W. J. Brackenbury. Therapeutic potential for phenytoin: targeting nav1.5 sodium channels to reduce migration and invasion in metastatic breast cancer. Breast Cancer Research and Treatment, 134(2):603-615, 2012.

33. H. Yosihara. Gravity waves on the free surface of an incompressible perfect fluid of finite depth. Publ. Res. Inst. Math. Sci., 18(1):49-96, 1982. 